\title{
Measuring the Thermodynamic Cost of Timekeeping
}

\author{
A. N. Pearson, ${ }^{1, *}$ Y. Guryanova, ${ }^{2, *}$ P. Erker $\oplus^{2,{ }^{*}}$ E. A. Laird $\odot,{ }^{3}$ G. A. D. Briggs $\odot,{ }^{1}$ M. Huber $\oplus^{2,4, \dagger}$ and N. Ares $\oplus^{1, *}$ \\ ${ }^{1}$ Department of Materials, University of Oxford, Oxford OX1 3PH, United Kingdom \\ ${ }^{2}$ Institute for Quantum Optics and Quantum Information (IQOQI), \\ Austrian Academy of Sciences, A-1090 Vienna, Austria \\ ${ }^{3}$ Department of Physics, Lancaster University, Lancaster, LA1 4YB, United Kingdom \\ ${ }^{4}$ Vienna Center for Quantum Science and Technology, Atominstitut, TU Wien, 1020 Vienna, Austria
}

(Received 23 June 2020; revised 20 January 2021; accepted 31 March 2021; published 6 May 2021)

\begin{abstract}
All clocks, in some form or another, use the evolution of nature toward higher entropy states to quantify the passage of time. Because of the statistical nature of the second law and corresponding entropy flows, fluctuations fundamentally limit the performance of any clock. This suggests a deep relation between the increase in entropy and the quality of clock ticks. Indeed, minimal models for autonomous clocks in the quantum realm revealed that a linear relation can be derived, where for a limited regime every bit of entropy linearly increases the accuracy of quantum clocks. But can such a linear relation persist as we move toward a more classical system? We answer this in the affirmative by presenting the first experimental investigation of this thermodynamic relation in a nanoscale clock. We stochastically drive a nanometer-thick membrane and read out its displacement with a radio-frequency cavity, allowing us to identify the ticks of a clock. We show theoretically that the maximum possible accuracy for this classical clock is proportional to the entropy created per tick, similar to the known limit for a weakly coupled quantum clock but with a different proportionality constant. We measure both the accuracy and the entropy. Once nonthermal noise is accounted for, we find that there is a linear relation between accuracy and entropy and that the clock operates within an order of magnitude of the theoretical bound.
\end{abstract}

DOI: $10.1103 /$ PhysRevX.11.021029

\section{INTRODUCTION}

By modern standards, the accuracy with which we can keep time is truly astonishing; nowadays the best atomic clocks keep time to an accuracy of approximately one second in every $10^{8}$ years [1]. This is more accurate than any physical constant we have ever measured (for example, the magnetic moment of an electron $g$ is known to 12 digits [2]), and better than computer arithmetic which has an accuracy of 16 digits for 64-bit calculations [3]. Atomic clocks run by the rules of quantum mechanics, targeting a specific hyperfine transition in an atom's energy spectrum; yet despite the great progress in keeping time, surprisingly little is known about the relation between quantum clocks and thermodynamics. Famously invariant under time

\footnotetext{
*These authors contributed equally to this work.

†marcus.huber@univie.ac.at

natalia.ares@materials.ox.ac.uk
}

Published by the American Physical Society under the terms of the Creative Commons Attribution 4.0 International license. Further distribution of this work must maintain attribution to the author(s) and the published article's title, journal citation, and DOI.
Subject Areas: Condensed Matter Physics, Statistical Physics reversal, the equations of quantum mechanics provide little explanation for the passage of time, whereas the theory of thermodynamics, although elucidating little more on the same front, does at least leave some entropic signatures [4-9]. One of the milestones at the intersection of the two fields is to derive a quantitative relation between the second law of thermodynamics and the flow of time. Investigations in this direction are also a vital component in our understanding of quantum thermodynamics, a field focused on the investigation, analysis, and design of machines on the quantum scale, to which clocks are no exception [10,11].

Alongside philosophical and conceptual curiosities, clocks constitute an intrinsic component in the operation of numerous systems, from the clocks used to time the gates on a desktop CPU to the clocks necessary for determining your GPS coordinates. In the quantum regime, as opposed to the classical case, the thermodynamic cost associated with the precise control of a system is comparable to the energy scale of the system itself [12-14].

For example, the cycles of a quantum Otto engine need to be controlled by a microscopic autonomous clock $[15,16]$, a device that produces a stream of ticks without any timing input or external control. The energetic cost of 
running this clock is comparable to the energetic output of the engine and thus can no longer be neglected. These clocks have been studied rigorously from the perspective of open quantum systems, where it has been shown that their performance with respect to the resources they consume is subject to particular relations, as well as trade-offs [4].

One of the challenges in deriving such relations from microscopic thermodynamic principles is that reasonably large systems are required for irreversible dynamics to emerge [17,18]. In developing relations and trade-off models, we are forced to make assumptions about the underlying parameters and system dynamics $[10,19]$.

At the other end of the scale, in the classical domain, it is difficult to keep track of thermodynamic costs because the systems become large and complex. Systems for which thermodynamic costs could be accurately estimated allowed for experimental breakthroughs in the study of stochastic and quantum thermodynamics. For example, a Brownian Carnot engine [20] and a single-atom heat engine [21] were demonstrated using electric field noise as a hot reservoir.

In this article, we experimentally explore the thermodynamic costs of timekeeping by directly measuring both the accuracy and the entropy generation associated with a simple nanoelectromechanical clock. In our implementation, the clock is a thermal engine that operates between a hot reservoir realized by means of a noisy electric field coupled to a mechanical element and a cold reservoir realized by a room-temperature measurement circuit. The clock is driven by the heat flow through the engine and the work required to observe the mechanical vibrations. The thermodynamics of clocks can thus be experimentally investigated, in the same way as conventional heat engines, by comparing the heat flow through the clock with the resulting accuracy. We compare our results to quantum and classical clock models, and analyze the relation of our findings to thermodynamic uncertainty relations (TURs) [6,22-25], arising in classical stochastic thermodynamics. In the latter, we find our results to be both conceptually and quantitatively different.

A clock, like any thermodynamic machine, operates by consuming a resource and creating waste in the form of entropy [Fig. 1(a)]. Its useful output is a train of ticks which can be counted by a register. Previously, two avenues of research in that direction have led to similar conclusions: on the one hand, using TURs, the authors of Ref. [26] show an entropic cost to measuring time based on a finite Markov model, on the other hand, a simple two-qubit quantum

\section{Clockwork}

\section{Register}

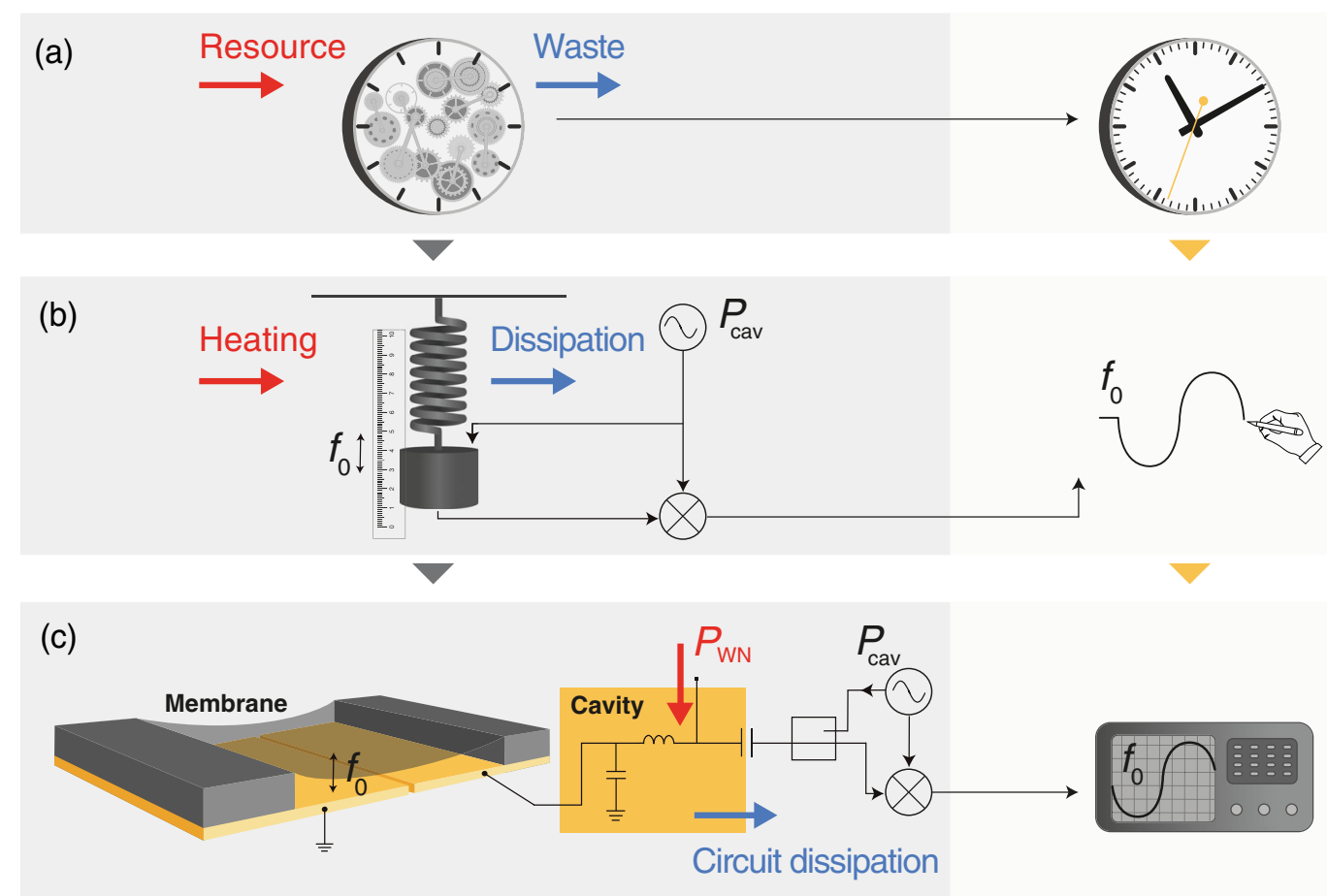

FIG. 1. (a) For timekeeping, the clockwork consumes resources, part of which are lost as waste. The hands of the clock register the clock's ticks. (b) Simple mechanical clock. Here, a mass is suspended from a spring and the heat from the environment excites the mass' motion at frequency $f_{0}$. These vibrations are probed by a signal of power $P_{\text {cav }}$. This system (the clockwork) generates a periodic signal, which is registered to identify the clock's ticks. (c) A schematic of our electromechanical system acting as a clock. A nanometer-thick membrane is driven by a white-noise signal of power $P_{\mathrm{WN}}$. The membrane's vibrations are probed by a rf cavity driven with a signal of power $P_{\text {cav }}$. The cavity output signal, and thus the clock tick's, are registered by an oscilloscope. 
engine coupled to a ladder was used to derive a similar entropic cost in autonomous quantum clocks [4]. Both of these show that in their respective regimes of validity there is a fundamental price to timekeeping: the more regular and frequent the ticks, the greater the rate at which the clock must create entropy.

This work experimentally and theoretically studies a new kind of classical clock which realizes this thermodynamic process. The clock is based on a simple optomechanical model [Fig. 1(b)], in which the Brownian motion of a mechanical resonator is monitored using an electronic cavity interferometer. Each mechanical oscillation identified by the interferometer corresponds to one tick. The clock is driven by the work performed to illuminate the cavity and by the heat transferred from the hot resonator to the cold measurement electronics. While the accuracy can be improved by increasing either the mechanical amplitude or the electrical illumination power, in both cases this leads to greater heat dissipation and therefore increased entropy, as explained in Sec. II and Appendix B.

The experimental realization of the model is shown in Fig. 1(c). The mechanical resonator is a high-quality silicon nitride membrane vibrating in its fundamental flexural mode. To excite quasi-Brownian motion, the membrane is driven by a white-noise electrical signal, which acts as an effective thermal bath that raises the mechanical mode temperature [27]. To monitor the membrane's displacement, it is capacitively coupled to a radio-frequency (rf) cavity operated in an optomechanical readout circuit [28-33]. The voltage output of this circuit is proportional to the instantaneous displacement. This output is recorded using an oscilloscope which acts as the clock register. Each completed oscillation, identified by an upward zero crossing of the voltage record, represents one tick of the clock.

We used our setup to test the relation between the resources used to power the clock and its accuracy. The accuracy was determined by an algorithm which marked the instance at which a tick (a particular behavioral signature of the membrane's motion) occurred. We then looked at the accuracy of the optomechanical system for a range of white-noise driving power and compared it to the prediction of a classical clock model. In order to make this comparison, we associated the system's resources to the clock's total entropy production. Our results confirm clear proportionality between the driving power (the resource) and the periodicity of the cavity output signal (the accuracy), which is the trademark response predicted by both a quantum and a classical clock model. This finding suggests that fundamental relations for the thermodynamics of timekeeping can be observed in a broad class of operating regimes, making them universal. In this way, our results support the idea that entropy dissipation is not just a prerequisite for measuring time's passage, but that the entropy dissipated by any clock is quantitatively related to the fundamental limit on that clock's performance.

\section{THEORY: THE THERMODYNAMIC COST OF TIMEKEEPING}

Be it quantum or classical, we define the accuracy of a clock as in Ref. [4]:

$$
N:=\left(\frac{t_{\text {tick }}}{\Delta t_{\text {tick }}}\right)^{2}
$$

where $t_{\text {tick }}$ is the mean interval between successive ticks and $\Delta t_{\text {tick }}$ is the standard deviation of this interval. Equivalently, $N^{-1}$ is the Allan variance [34] when the observation period is equal to $t_{\text {tick }}$. This is a more severe measure of accuracy than the Allan variance of a much larger number of ticks. If Markovian stationarity is assumed, i.e., if successive tick intervals are uncorrelated, $N$ is also the number of ticks before the expected accumulated timekeeping error is equal to one tick interval.

Our objective is to test the measured value of $N$, derived by analyzing a series of ticks generated by the experiment, against the prediction of models in which the accuracy of the clock appears as a function of the resources used to drive it. This line of inquiry is inspired by Ref. [4], in which the rate of entropy production and accuracy of an autonomous quantum clock are found to be linearly related (assuming weak coupling), i.e.,

$$
N_{Q}=\frac{\Delta S_{\text {tick }}}{2 k_{B}}
$$

where $k_{B}$ is Boltzmann's constant and $\Delta S_{\text {tick }}$ is the entropy generated per tick. This entropy arises due to power being dissipated by the clock, from which we understand that greater power dissipation corresponds to greater accuracy.

In similar spirit we have analyzed a classical model of the optomechanical experiment of Fig. 1(c).

In this optomechanical clock, the electrical cavity containing the membrane is excited with a cavity illumination tone with power $P_{\text {cav }}$. Part of this tone is reflected from the cavity, and its phase is modulated by the thermal vibration of the membrane. By identifying each modulation cycle, a series of ticks can be derived, repeating at a interval set by the mechanical period. However, there is a thermodynamic price. For accurate timekeeping, the modulated signal should be made as large as possible. This increases the dissipation in the detection circuit, and therefore creates greater entropy than a small signal. By calculating the minimal uncertainty with which ticks can be identified in the presence of thermal noise, Appendix B shows that the greatest possible accuracy for this classical clock is

$$
N_{C}=\frac{2 \pi^{2}}{k_{B}} \frac{T_{c}}{T_{N}} \Delta S_{\text {tick }}
$$

where $T_{N}$ is the noise temperature of the measurement electronics and $T_{c}$ is the temperature of the environment, 
assumed to be colder than the mechanical effective temperature.

Increasing the thermodynamic resources supplied to the clock, i.e., $P_{\text {cav }}$ and $P_{\mathrm{wN}}$, increases both the accuracy and the entropy creation rate. Intriguingly, this classical experiment, despite representing a completely different physical system from the quantum clock of Ref. [4], obeys a similar relationship between accuracy and entropy. Whereas $N$ in Eq. (1) is the accuracy which can be extracted from a sequence of ticks experimentally realized by the clock, $N_{C}$ in Eq. (3) is a statistical prediction based on the thermodynamic properties of the setup.

In order to compare the values of $N$ obtained from the experiment with the prediction of the model, we must identify the source of entropy $\Delta S_{\text {tick }}$ in our system. We acknowledge there are various types of entropy emerging from the setup. Here, we focus on the entropy in the cavity output signal, as it is directly observable in our temporal traces. Additional entropy contributions are of course produced in the instruments used to control the systems (from the tone that drives the readout cavity to the oscilloscope that measures the cavity output signal). We do not focus on this type of entropy, as it depends on the specific implementation and it is not present in autonomous devices. Finally, there is the source of entropy production that comes from the white-noise driving. This is the fundamental entropy dissipated per natural temporal event (tick) in our experiment. Here it is important to note that not all of the power injected in the system will be converted into a useful drive signal, just as not all the energy from a hot bath can be converted into work in a heat engine; some will be dissipated in the environment at the expense of entropy production elsewhere. This does not impact our results as long as the power of the white-noise signal used to drive the clock is high enough to make the ticks identifiable above the thermal background. Thus, we estimate the relevant entropy $\Delta S_{\text {tick }}$ from the spectral density of the cavity output signal by computing the area of the spectral density peak located at the membrane's resonance frequency.

\section{EXPERIMENTAL SETUP}

The vibrating membrane is measured using the setup shown in Fig. 2(a). The membrane, which consists of $50 \mathrm{~nm}$ thick SiN metallized with $\mathrm{Al}$, is suspended over two $\mathrm{Cr} / \mathrm{Au}$ electrodes patterned on a silicon chip, forming a capacitor. A dc voltage $V_{\mathrm{dc}}=15 \mathrm{~V}$ is applied to electrode 1 , with electrode 2 grounded. Electrode 1 is connected to a rf cavity, which is realized with an inductor and capacitors [Fig. 2(a)]. As the membrane vibrates, the capacitance $C_{C}$ between the membrane and the electrodes changes. Driving the rf cavity with a resonant tone, we can probe the membrane's motion by monitoring the cavity's output signal [33]. The cavity is driven by injecting a rf signal via port 1 via a directional coupler. A signal to excite the membrane's motion is incorporated in the circuit via port 3 .

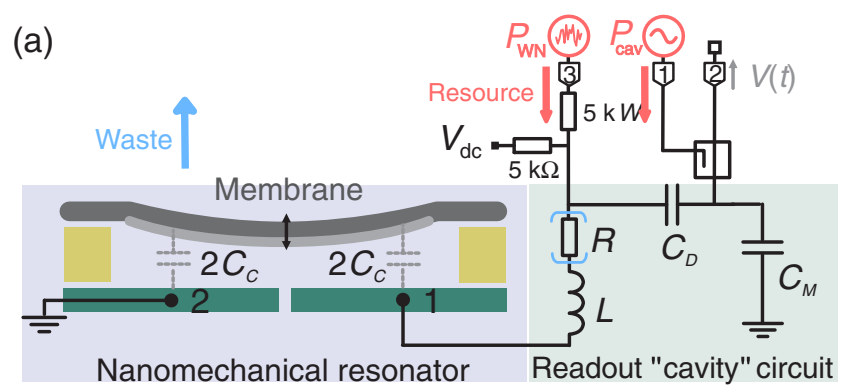

(b)

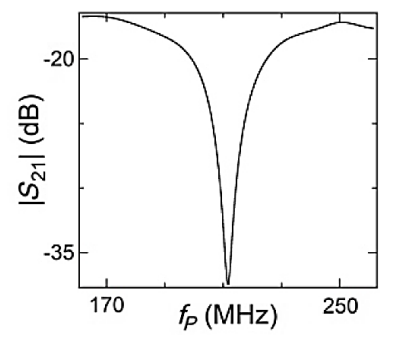

(c)
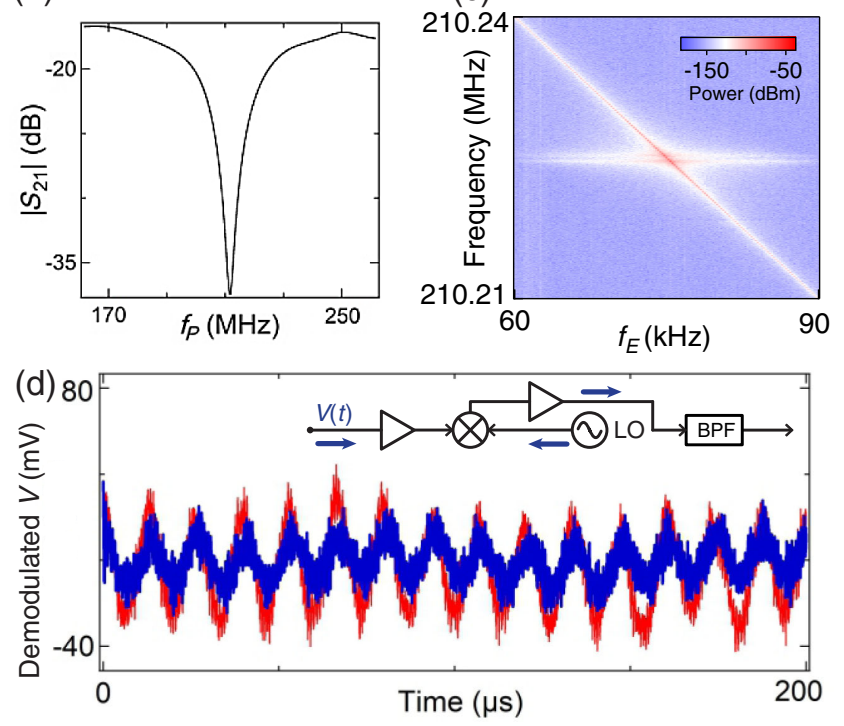

FIG. 2. (a) Experimental setup. A metalized silicon nitride membrane is suspended over two metal electrodes, forming a capacitor $C_{C}$. One of the electrodes is connected to a rf tank circuit which acts as a readout cavity. Electrode 2 is grounded. The tank circuit is formed from a $223 \mathrm{nH}$ inductor $L$, and two $10 \mathrm{pF}$ capacitors $C_{D}$ and $C_{M}$. Parasitic capacitances contribute to $C_{M}$ and parasitic losses in the circuit are parametrized by an effective resistance $R$. The cavity can be probed by injecting a rf signal at port 1 via a directional coupler. The output signal is measured at port 2 using a vector network analyzer or a spectrum analyzer. The membrane's motion can be excited by injecting a signal at port 3 . Bias resistors allow a dc voltage $V_{\mathrm{dc}}$ to be applied to electrode 1 . Red (blue) arrows indicate resources (waste) for our system. (b) $\left|S_{21}\right|$ as a function of probe frequency $f_{p}$. (c) One of the mechanical sidebands observed in the spectrum of the cavity output signal when an excitation tone at frequency $f_{E}$ is injected at port 3 and swept in frequency while the cavity is driven at its resonant frequency via port 1 . The sideband power grows when $f_{E}$ coincides with the resonance frequency of the membrane $f_{0}$. (d) Demodulated readout signal $V(t)$, as a function of time, for $P_{\mathrm{cav}}=14 \mathrm{dBm} . P_{\mathrm{WN}}=0.25 \mathrm{~W}$ and $P_{\mathrm{WN}}=0.063 \mathrm{~W}$ for the red and blue traces, respectively. The inset shows the demodulation circuit. (LO, local oscillator; BPF, bandpass filter).

The experiment is carried out at room temperature at approximately $5 \times 10^{-6}$ mbar.

To determine the cavity's resonant frequency, we measure the scattering parameter $\left|S_{21}\right|$, which is proportional to the reflection from the cavity, as we sweep the frequency of 
a probe tone $f_{P}$. The cavity resonance is evident as a minimum in $\left|S_{21}\right|$ [Fig. 2(b)]. To identify the mechanical resonance, we perform two-tone spectroscopy. While driving the cavity at its resonance frequency (i.e., with $f_{P}=210.3 \mathrm{MHz}$ ) through port 1, we applied another tone of frequency $f_{E}$ through port 3 in order to excite the membrane. The power spectrum of the reflected signal is shown in Fig. 2(c) as a function of $f_{E}$. The mechanical response is evident as a strong increase in the sideband power at $f_{P} \pm f_{E}$ when $f_{E}$ matches the mechanical frequency $f_{0} \approx 74.5 \mathrm{kHz}$ [33].

In order to use the membrane as a thermal clock, we drive the membrane's motion (of the fundamental mode) stochastically by applying a white-noise signal of power $P_{\mathrm{WN}}$ and bandwidth $500 \mathrm{kHz}$ through port 3 . This white-noise signal is the clock's heating resource. To register the ticks, we must illuminate the cavity, and to do this the resource is a resonant drive tone injected through port 1 with power $P_{\text {cav }}$. We measure the displacement of the membrane in real time by demodulating the cavity output signal $V(t)$. The demodulated signal is measured with an oscilloscope. We show $V(t)$ after demodulation and amplification for two different values of $P_{\mathrm{WN}}$ in Fig. 2(d). From these time traces, the ticks of the clock can be identified, and an accuracy can be computed for different values of $P_{\mathrm{WN}}$.

Studying clock performance in the absolute sense is not strictly possible in our system, since this would require us to synchronize multiple clocks (e.g., via the alternating ticks game [5,35]). We have thus chosen a reference clock that is orders of magnitude faster than the system under investigation in order to resolve the temporal dynamics. In our case, the membrane's frequency is in the kilohertz regime while our reference clock, the clock of the oscilloscope, operates at a frequency several orders of magnitude higher. Our system constitutes a quasi-autonomous clock, since just with a driving tone, it is able to convert the power of the white noise driving the membrane's motion into the observable ticks of a clock.

\section{RESULTS}

Ticks are generated from time records of the demodulated voltage signal as shown in Fig. 2. Each tick corresponds to an upward zero crossing of this signal. In principle, these zero crossings could be identified in nearly real time using a threshold detector with an appropriate input filter. In practice, we acquired the entire voltage record and identified ticks in postprocessing, in order to be able to study the effects of different filter and threshold settings.

At each setting of $P_{\mathrm{cav}}$ and $P_{\mathrm{WN}}$, a record of raw data with a duration of $1 \mathrm{~s}$ was stored. In order to suppress noise, each record was then digitally filtered using a bandpass filter of $75 \mathrm{kHz}$ bandwidth centered at $f_{0}$. This bandwidth, which is nearly equal to $f_{0}$, is sharp enough to remove much of the electronic noise, and thus avoids triggering false upward zero crossings, but has a fast enough ringdown to ensure that successive ticks are nearly independent. In a real-time clock, it could be implemented using an analog filter. To extract $N$ for each record, the upward zero crossings were identified in order to generate a sequence of tick intervals, and the resulting standard deviation $\Delta t_{\text {tick }}$ was substituted into Eq. (1).

The results of this analysis are shown in Fig. 3(a) as a function of $P_{\text {cav }}$ and $P_{\mathrm{WN}}$. For small values of $P_{\mathrm{WN}}$, we see that $N$ increases approximately linearly with $P_{\mathrm{WN}}$. This can be understood intuitively: a stronger drive makes the mechanical oscillations easier to distinguish from the noise. As $P_{\mathrm{WN}}$ increases further, the linear relationship breaks down and the accuracy shows signs of saturating. This is to be expected due to noise in the circuit leaking from the
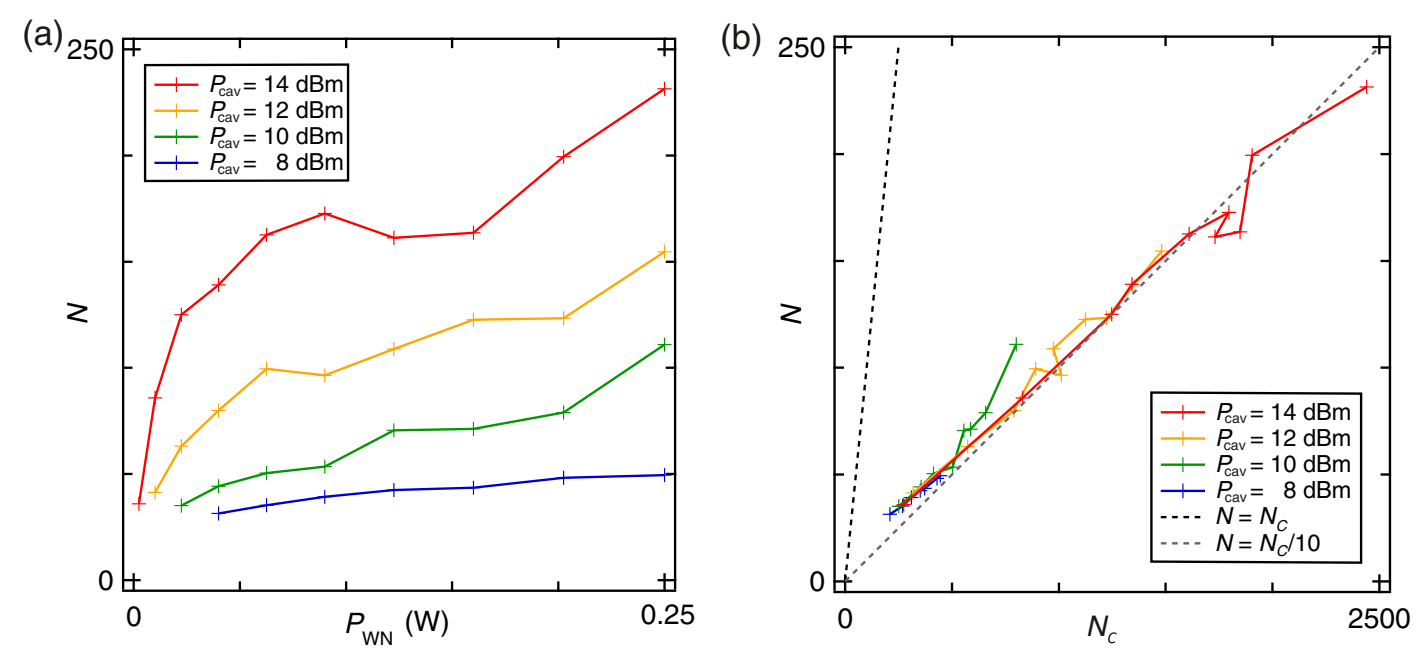

FIG. 3. (a) Accuracy $N$ versus white-noise power $P_{\mathrm{WN}}$ for $P_{\text {cav }}$ in the range $8-14 \mathrm{dBm}$. (b) Accuracy $N$ versus the accuracy predicted from the classical model $N_{C}$ for $P_{\text {cav }}$ in the range $8-14 \mathrm{dBm}$. The black dotted line is a guide to the eye showing the slope corresponding to $N=N_{C}$. The gray dotted line is chosen to approximate the slope of the displayed curves. 
heating tone and the membrane's motion entering the nonlinear regime, effects which do not allow a continued increase of $N$.

As $P_{\text {cav }}$ increases, the linear increase of $N$ as a function of $P_{\mathrm{WN}}$ shows a larger gradient. This is because an increased $P_{\text {cav }}$ enhances readout. Above $P_{\text {cav }}=14 \mathrm{dBm}$, however, demodulated $V(t)$ shows significant fluctuations, leading to the saturation of $N$ at smaller values of $P_{\mathrm{wN}}$ (see Appendix D). The time traces corresponding to $P_{\text {cav }}<8 \mathrm{dBm}$ are too noisy for ticks to be identified (see Appendix E). The oscilloscope's sampling rate was $40 \mathrm{MSa} / \mathrm{s}$, giving a resolution of $25 \mathrm{~ns}$ to the acquired time traces. Given the frequency of the membrane, this resolution sets an upper limit to the measurable accuracy of $N \lesssim 290000$; however, as seen from Fig. 3(a), experimental values of $N$ are less than a hundredth of this limit.

To test the predictions of the classical clock model, we now compare the measured $N$ with the predicted accuracy $N_{C}$ according to Eq. (3). The relevant entropy arises from the electrical power dissipated in the amplifier circuit by the optomechanical sidebands that contain the displacement information. As shown in Appendix B, the ratio $\Delta S_{\text {tick }} / T_{N}$ can be calculated from the same demodulated voltage record used to identify ticks. To do this, each record is first numerically transformed to generate a power spectrum. The entropy $\Delta S_{\text {tick }}$ is then calculated from the integrated power within a $10 \mathrm{kHz}$ window centered on the signal frequency $f_{0}$; the noise temperature $T_{N}$ is calculated from the average spectral density well away from this frequency [see Eq. (B43)]. The physical temperature of the measurement circuit is taken as $T_{c}=300 \mathrm{~K}$.

We have compared the values obtained for $N_{C}$ with the accuracy $N$ computed as in Eq. (1) [Fig. 3(b)]. Our results confirm that increasing accuracy requires increasing $\Delta S_{\text {tick}}$, and show the linear relation predicted by Eq. (3). However, the constant of proportionality, for all heating and illumination powers shown here, is approximately 10 times smaller than predicted. Since Eq. (3) represents an upper bound on the clock's efficiency, this discrepancy is not inconsistent with the theory. It probably indicates that identifying the zero crossings, which does not use all the information in the voltage record, is not an optimal procedure for identifying ticks. An improved tick identification algorithm might allow us to get closer to the bound set by the classical model of our setup.

\section{DISCUSSION}

Our experiment is simple enough to account for the thermodynamic resources used, like in Ref. [36], and at the same time our system is too complex to be modeled by a simple open quantum systems approach.

The results in Fig. 3 showcase an important relation between the accuracy and the entropy production that should be present in the most fundamental clocks [4], both in a quantum and a classical model. The accuracy is only a lower bound on the entropy creation, making it entirely possible for the system to dissipate more entropy at higher drive powers without providing more accurate ticks. The fact that we nonetheless see such a consistent linear relation between the accuracy and the entropy production for a considerable range of cavity and white-noise drives indicates that our clock's performance is close to optimal and that we are correctly identifying the relevant entropy contributions.

Our clock provides a steady stream of ticks that are identified from cumulative events; it would defeat the purpose of a clock if only a finished sequence of events can be used retroactively for the identification of ticks. That would rather correspond to the concept of a stopwatch, where upon interrogation one obtains a good estimate of how much time has elapsed between initialization and interrogation, but does not provide a continuous temporal reference frame. Although the system is not fully autonomous, because a cavity drive is necessary for readout, it presents a perfect test bed for generating a stable timeordered signal by exploiting thermal nonequilibrium. In fact, any system that acts as a register is expected to consume work, as it would inevitably require to perform measurements of irreversible events [37].

Any thermally irreversible process could be used as a clock [7], e.g., simply by observing the progress of equilibration as a proxy for time. We propose that an operational definition for a good clock is a system that reduces the linear slope of the accuracy-dissipation relation and keeps it linear for accuracy as high as possible. This is consistent with another recent finding Ref. [9], which shows that clockwork complexity can be used to decrease that linear slope and to increase the saturation point, beyond which extra dissipation will not correspond to a better clock quality.

The observed relationship between drive power and accuracy (Fig. 3) is in qualitative agreement with the relation stemming from the oversimplified model in Ref. [4], and with the prediction of our classical model. Our results also corroborate the notion that the quality of the arrow of time is indeed limited by the entropy dissipated by a clock. As described in Ref. [4], the linear relation between accuracy and entropy production tends to break down at some point. We have observed this effect in our experiment, most likely due to the membrane's motion entering the nonlinear regime at high drive powers or due to other nonlinearities playing a more significant role in the circuit. Below that threshold, our observed relationship between drive power and accuracy points toward a universal relation, in both quantum and classical regimes, between entropy production and clock accuracy.

We also note an interesting relation to the phenomenon of stochastic resonance [38], where noise can push a signal beyond a detection threshold and in this way increase the signal quality. Superficially our experiment is a similar scenario, since we inject noise to create a periodic signal. 
However, instead of using noise to make a preexisting signal detectable, this experiment uses noise to create the signal by heating the mechanical mode. Nevertheless, it will be interesting to see if these techniques can be fruitfully adapted to our setup.

We now consider the connection between these results and the thermodynamic uncertainty relations. These relations are a large class of inequalities that apply to out-ofequilibrium systems and relate the fluctuations of a driven observable $F$, such as the rate of a chemical reaction, to the entropy created $[6,22-25]$. They have the form

$$
\begin{aligned}
\mathfrak{p}(F) & \equiv \frac{|\langle F\rangle|^{2}}{\operatorname{Var}(F)} \\
& \leq g(\dot{S}),
\end{aligned}
$$

where $\mathfrak{p}(F)$ is known as the precision and $g(\dot{S})$ is a monotonic function of the entropy creation rate $\dot{S}$. [We follow previous literature by calling $N$ as in Eq. (1) the accuracy [4] and $\mathfrak{p}$ as in Eq. (4) the precision [25], even though these do not correspond to the usual definitions of accuracy and precision in metrology.] A relation such as Eq. (5) describes a trade-off between precision and dissipation. To achieve high precision by overwhelming the observable's thermal fluctuations, the system must be strongly driven and this creates entropy. An example of an experimental verification of TURs can be found in Ref. [39].

One should add that while the upper bound on precision $g(\dot{S})$ increases with increased dissipated entropy, that by no means implies a generically increased precision in more strongly driven systems. Indeed, for canonical thermodynamic choices in observables $F$, the precision of our system actually decreases with an increased drive. One way to view the relation of clock accuracy with increased entropy dissipation through the lens of TURs would be to identify the output of our tick identification algorithm in relation to a reference clock with the observable in a TUR; i.e., $\left\langle F_{\text {tick }}\right\rangle=t_{\text {tick }}$. While this is not really a standard observable as its very definition is dynamically updated, one can easily see that in this case, the accuracy of the clock is equal to the precision defined in the TUR.

So from that point of view, the noncanonical observable can be taken as the measured interval between successive ticks. If such an identification is possible, the combination of Eqs. (1) and (3) leads to the following relation:

$$
\mathfrak{p}\left(F_{\text {tick }}\right)=N \leq 2 \pi^{2} t_{\text {tick }} \frac{T_{c}}{T_{N}} \frac{\dot{S}}{k_{B}},
$$

which clearly has the form of Eq. (5).

Indeed, previous work [6,22-25] has shown that with an appropriately chosen observable, the precision satisfies a TUR inequality, which in the case of this work is given by the above. However, we emphasize that calculating the accuracy of this experimental clock is not simply a matter of applying known TURs.

Equation (6) is useful only if there is a mechanism to identify ticks and therefore correctly measure the observable. Naively applying Eq. (4) to the wrong observable, even if that observable is derived from the same voltage record as that used to generate ticks, leads to a precision that can be very different from the accuracy as defined by Eq. (1). In fact, with the wrong observable, running the clock at a higher entropy generation rate can actually lead to a worse precision.

To see this surprising fact, suppose we take the observable $F$ as the energy flux contained in the demodulated output of the clock, averaged over a fixed interval. When the clock is weakly driven, this output consists of broadband voltage noise which carries energy at a nearly constant rate and therefore leads to a large precision defined by Eq. (4). When the clock is strongly driven, the output is dominated by the mechanical signal, which has a long correlation time and therefore induces long-lived fluctuations in the flux.

Let us now demonstrate this result quantitively. We select as an observable the power entering the measurement circuit, averaged over an interval chosen as 10 cycles of the mechanical oscillation. This is a natural choice to make, because the observable is conventionally taken as a current [39]. In this case it is the energy current into the measurement circuit. Up to a proportionality constant, which cancels from Eq. (4), this energy is

$$
F(t)=\frac{1}{10 t_{\text {tick }}} \int_{t}^{t+10 t_{\text {tick }}} V^{2}\left(t^{\prime}\right) d t^{\prime},
$$

where $V(t)$ is the demodulated voltage record, as plotted, e.g., in Fig. 2(d).

Figure 4 shows the energy precision calculated from Eqs. (4) and (7) from the same demodulated voltage record as in Figs. 3(a) and 10(a). As can be seen, the precision $\mathfrak{p}(F)$ of this observable differs drastically from the accuracy $N$, and even has the opposite dependence on both $P_{\mathrm{WN}}$ and $P_{\text {cav }}$. The reason is that the slowly fluctuating mechanical signal delivers a strongly varying amount of power [i.e., low precision, defined by Eq. (4)] but nevertheless encodes a regular time step [and therefore high accuracy, defined by Eq. (1)].

To compare this data with the classical clock model, Appendix $\mathrm{C}$ presents an analytic derivation for the thermodynamic precision of our experiment in terms of this $F$. We obtain

$$
\mathfrak{p}(F)=\left(1+\frac{\alpha}{P_{\mathrm{cav}} P_{\mathrm{WN}}}\right)^{2},
$$

where $\alpha$ is a constant proportional to the intensity of the measurement noise. This equation is used as a fitting 


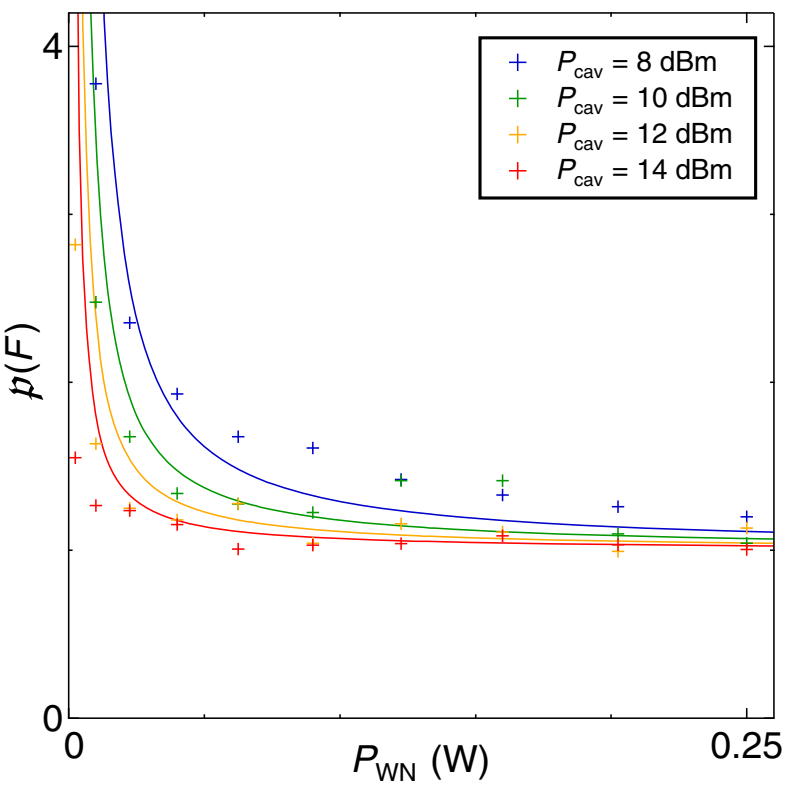

FIG. 4. Precision $\mathfrak{p}(F)$ of the demodulated signal as a function of mechanical drive power $P_{\mathrm{WN}}$ for different settings of the cavity illumination power $P_{\text {cav }}$. Crosses show precision calculated from the voltage record data using Eqs. (4) and (7). The experimental data are consistent with the limiting cases of the theory. Curves show predictions from Eq. (C31). The free parameter $\alpha$ is set by fitting to the data with $P_{\text {cav }}=8 \mathrm{dBm}$, then held fixed to generate the other curves. We separate the data into two figures for cavity drive powers $P_{\text {cav }}$ of $8-14 \mathrm{dBm}$ (for powers $16-20 \mathrm{dBm}$, see Appendix C).

function in Fig. 4, with $\alpha$ as the fit parameter. We fit the data for $P_{\text {cav }}=8 \mathrm{dBm}$ to extract the value $\alpha=8.6 \times 10^{-5} \mathrm{~W}^{2}$. This value of $\alpha$ was then used to predict $\mathfrak{p}(F)$ curves in Fig. 4 for all the other datasets, with no free parameters.

This analysis shows that the clock output encodes a temporal signal in a way that is not apparent in the chosen observable, despite the fact that energy flux given by Eq. (6) is at first sight a reasonable choice as the basis of a clock. This shows that although TURs are useful bounds in a wide range of systems, they must be applied with care even in a fairly elementary clock realization such as this one.

\section{CONCLUSION AND OUTLOOK}

In this work, we demonstrated a thermomechanical clock which allowed us to reveal a universal relation in the thermodynamics of timekeeping. We first showed that the heating resource introduced to drive the clockwork of our optomechanical setup enhances the accuracy of the clock signal. Modeling our system classically, we then found that the linear relationship between clock accuracy and entropy production, derived in an idealized quantum setting, is found to hold in the classical regime. The universality of this relation provides a clear link between the entropy dissipated by the clock and the quality of the arrow of time. We also uncover an interesting relation to thermodynamic uncertainty relations studied in the field of stochastic thermodynamics. In some sense, our method of identifying ticks can be interpreted as a means to finding observables that maximally increase precision with increased entropy dissipation rates. We showed that in our system, contrary to the identified clock ticks, conventional thermodynamic currents do not experience an increased precision with increased driving. As all clocks are fundamentally thermodynamic in nature $[8,9]$, we believe that a further study of the relation between TURs and clocks will be fruitful.

Another exciting avenue for future investigation that one can imagine would be interpreting the system as a heat engine, instead of a clock. Since the oscillations of the membrane can induce a current, they are able to produce work, thus mimicking a heat engine that converts unstructured noise into regular beats. For a system of this scale, work fluctuations become crucial, in contrast to a classical macroscopic engine, for which the power delivered in each stroke is approximately the same. This opens up the opportunity of studying work fluctuation relations as well as deriving rates for heat to work conversion. Finally, it would be interesting to see if the noise (heat) driving the membrane could be harnessed from the environment, rather than being input from a characterized source; in this way one would be able to say that the system is truly performing as a useful engine.

\section{ACKNOWLEDGMENTS}

We acknowledge useful discussions with G. Milburn, M. Lock, M. Schwarzhans, and J. Parrondo, as well as F. Vigneau's contribution to the experiment. This work was supported by the Royal Society, EPSRC Platform Grant (EP/R029229/1), Grant No. FQXi-IAF19-01 from the Foundational Questions Institute Fund, a donor advised fund of Silicon Valley Community Foundation, and by the European Research Council (ERC) under the European Union's Horizon 2020 research and innovation programme (Grant Agreements No. 818751 and No. 948932). This publication was also made possible through support from Templeton World Charity Foundation. The opinions expressed in this publication are those of the authors and do not necessarily reflect the views of the Templeton Foundations. M. H. and P.E. acknowledges funding from the Austrian Science Fund (FWF) through the START project Y879-N27, FQXi Grant (No. FQXiIAF19-03-S2), and the ESQ Discovery Grant Emergent time-operationalism, quantum clocks and thermodynamics of the Austrian Academy of Sciences (ÖAW). P. E. and Y. G. acknowledge funding from the Austrian Science Fund (FWF) through the Zukunftskolleg ZK03.

\section{APPENDIX A: ELECTROMECHANICAL SYSTEM}

The silicon nitride membrane is $50 \mathrm{~nm}$ thick and has an area of $1.5 \times 1.5 \mathrm{~mm}^{2} .90 \%$ of the area of the membrane is 
metalized with $20 \mathrm{~nm}$ of $\mathrm{Al}$. We suspend this membrane over two $\mathrm{Cr} / \mathrm{Au}$ electrodes patterned on a silicon chip. The capacitor formed between the electrodes and the metalized membrane, which depends on the membrane's displacement, leads to coupling between the cavity and the mechanical motion. The rf circuit is modeled and characterized in Ref. [33]. The entire setup forms a three-terminal circuit with input ports 1 and 3 and output port 2 . We used a vector network analyzer to measure the scattering parameter [Fig. 1(b)], a spectrum analyzer to measure power spectra [Fig. 1(c)], and an oscilloscope to measure the displacement as a function of time [Fig. 1(d)].

\section{APPENDIX B: ENTROPY-ACCURACY RELATION FOR A THERMOMECHANICAL CLOCK}

Here, we derive the entropy-accuracy relation, Eq. (3), which is tested in the main text. We do this by considering two classical clock models. The first is a very simple clock that uses the filtered Johnson noise of a hot resistor. The second is the optomechanical clock-an elaboration of the Johnson-noise clock which is realized in our experimental setup. As shown below, both designs obey the same relation, which in turn resembles previously derived relations for classical [26] and quantum [4] clocks.

In both models, the clock must derive ticks from a periodic but noisy voltage record. We ask the question, how precisely can any clock identify a tick instant from a segment of this record? From the perspective of the clock, this is clearly a problem of phase estimation. From the $n$th segment of the record, an error $\delta \phi_{n}$ in estimating the phase leads to an error $\delta t_{n}=t_{\text {tick }} \delta \phi_{n} / 2 \pi$ in identifying the corresponding tick instant $t_{n}$. Thus, from Eq. (1), the clock accuracy in any classical model is related to the phase error by

$$
N_{M}:=\frac{4 \pi^{2}}{\left\langle\left(\delta \phi_{n}\right)^{2}\right\rangle}
$$

(a)

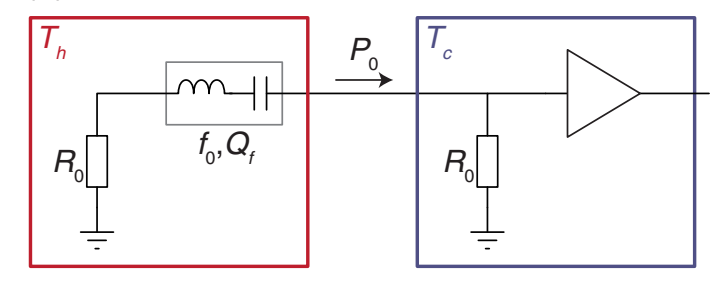

since the tick uncertainty $\Delta t$ is by definition the root-meansquare value of $\delta t_{n}$. Furthermore, we require that successive ticks be statistically independent, which means that every tick must be derived from a nonoverlapping segment of the record. In what follows, we construct models for $\delta \phi_{n}$ for two physical scenarios and thus estimate the accuracy of those clock models.

\section{Measuring time from filtered Johnson noise}

Figure 5 shows a design for a thermodynamical clock based on Johnson noise. The clock contains two heat baths at temperatures $T_{h}$ and $T_{c}$. Inside the hot bath, at temperature $T_{h}$, is a resistor $R_{0}$, which is connected via a matched transmission line to an ideal voltage amplifier located in the cold bath at temperature $T_{c}$. To ensure an impedance match and thus prevent reflections from the end of the transmission line, an equal resistor $R_{0}$ is connected to the amplifier input. A reflective bandpass filter is placed in the transmission line, centered at frequency $f_{0}$ and with quality factor $Q_{f}$, so that it passes frequencies in a bandwidth of $f_{0} / Q_{f}$ near the center frequency. The combined Johnson noise of the two resistors leads to an incoherent voltage oscillation at the cold amplifier input, whose peak amplitude $V_{S}$ satisfies

$$
\left\langle V_{S}^{2}\right\rangle=2 k_{B}\left(T_{h}+T_{c}\right) R_{0} \frac{f_{0}}{Q_{f}},
$$

where $\langle\cdot\rangle$ denotes an average over many oscillations. Each oscillation cycle corresponds to one tick of the clock. Demarcating each cycle accurately requires a large oscillation amplitude, meaning that a larger power is dissipated in the cold resistor; this is the thermodynamic price that we aim to quantify.

The amplifier measures the input voltage $V(t)$ as a function of time $t$ [Fig. 5(b)]. To generate a timing signal, the clock's task is to identify ticks from particular instances of the record, for example, those instants at which the upward crossings of the $t$ axis occur. This is the phase estimation problem described above. The reason that a (b)

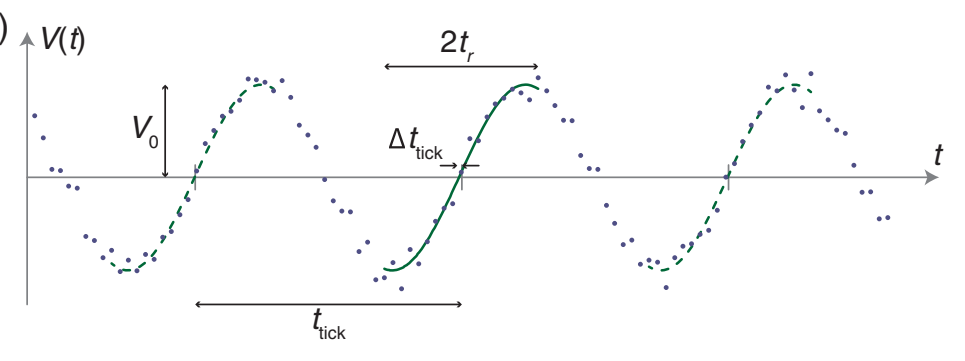

FIG. 5. Simple electrothermal clock. (a) The setup. The Johnson noise of resistor $R_{0}$, in equilibrium with a hot bath at temperature $T_{h}$, is filtered to pass frequency $f_{0}$ with bandwidth $f_{0} / Q_{f}$. The resulting signal, whose power is $P_{0}$, is passed to a matched resistor and amplifier at temperature $T_{c}$. (b) From the noisy voltage record (points) seen by the amplifier, we can generate clock ticks by estimating the zero crossing of each cycle using a sinusoidal fit (lines). Here $\Delta t$ marks the sampling interval, $t_{\text {tick }}=1 / f_{0}$ is the average tick interval, $\pm t_{r}$ is the fit range, and $\Delta t_{\text {tick }}$ is the fit uncertainty. 
perfect estimate is impossible even in principle is that the record is contaminated by voltage noise, including the broadband Johnson noise of the cold resistor.

How should the clock best perform a phase estimate, given a segment from the noisy voltage record? The answer is to perform a maximum-likelihood estimation. If the noise is uncorrelated and has a Gaussian distribution, as expected for broadband Johnson noise, this means a least-squares fit to the data [40]. No implementation of the clock can perform better than this.

To this end, we imagine that we have obtained some experimental data; we discretize the time interval in the record into pieces around the expected tick locations (the upward crossings), and fit one curve for each tick of the clock, such that for $n$ ticks we fit $n$ curves. For a particular tick we imagine fitting the function

$$
\begin{aligned}
& V\left(t \mid \phi_{n}\right)=V_{0} \sin \left(2 \pi f t+\phi_{n}\right) \quad \text { with } \\
& 2 n \times t_{\text {tick }}-t_{r} \leq t \leq 2 n \times t_{\text {tick }}+t_{r} \quad \text { for } n \in \mathbb{Z},
\end{aligned}
$$

where $V_{0}$ is the oscillation amplitude, $f$ is the frequency, $\phi_{n}$ is the phase, and where we have chosen to fit the $n$th tick to a function over the interval $2 t_{r}$ [see Fig. 5(b)]. The parameters $V_{0}$ and $f$ can be estimated over several recent oscillation cycles because they are slowly varying properties and are therefore not determined by the noise over a single cycle. The only parameter to fit is thus the phase $\phi_{n}$, which motivates the notation $V\left(t \mid \phi_{n}\right)$ as per the prescription in Ref. [40]. For a particular dataset $\mathcal{D}$, the optimal value of the parameter for the $n$th tick, denoted $\phi_{n}^{*}$, is the one that minimizes the $\chi^{2}$ function, defined as

$$
\chi^{2}\left(\phi_{n}\right)=\sum_{i}\left(\frac{V_{i}-V\left(t_{i} \mid \phi_{n}\right)}{\sigma_{i}}\right)^{2},
$$

where $i$ labels the data points and ranges over the total number of data points, and $\sigma_{i}$ is the vertical standard deviation of each point. The uncertainty is then determined by $\Delta \chi^{2}=1$ and the curvature parameter $\alpha$, and follows the expression

$$
\Delta \phi:=\sqrt{\left\langle\left(\delta \phi_{n}\right)^{2}\right\rangle}:=\sqrt{\Delta \chi^{2}} \alpha^{-1 / 2} .
$$

The curvature parameter is calculated from the fitted function and the experimental points $i$ as

$$
\alpha:=\sum_{i} \frac{1}{\sigma_{i}^{2}}\left(\frac{\partial V\left(t_{i} \mid \phi_{n}\right)}{\partial \phi_{n}}\right)^{2} .
$$

A final value for Eq. (B5) would be obtained by evaluating $\alpha$ at the fitted parameter $\phi_{n}^{*}$ which minimizes Eq. (B4) and choosing $\Delta \chi^{2}$ such that it corresponds to the desired confidence interval. Since we are in the business of constructing a model for the accuracy (i.e., we are not analyzing the fit of a particular dataset), we must make a statement that is reasonable for all datasets $\{\mathcal{D}\}$ that may emerge from this setup. To do this, we must make a few additional assumptions. First, we are interested in a situation where the oscillation frequency is sharply defined, i.e., $Q_{f} \gg\left\langle V_{0}^{2}\right\rangle / \sigma_{i}^{2}$, which means that within a single cycle $\sigma_{i}$ is dominated by the broadband noise at the amplifier input and therefore takes a constant value $\sigma$ for all data points. Next, we imagine that the $n$ ticks are fitted by choosing $n$ windows (or regions) of length $2 t_{r}$, where $t_{r}=1 / 2 f_{0}$, and the $\chi^{2}$ minimization gives us the value of the crossing $\phi_{n}^{*}$ for each tick. To calculate $\alpha$ in any such region, we idealize Eq. (B6) by imagining a continuum of data points, and thus convert the sum to an integral normalized by $\Delta t$, the sampling interval. This gives

$$
\begin{aligned}
\alpha & =\frac{1}{\sigma^{2} \Delta t} \int_{-t_{r}}^{t_{r}}\left(\frac{\partial V\left(t \mid \phi_{n}\right)}{\partial \phi_{n}}\right)^{2} d t \\
& =\frac{V_{0}^{2}}{\sigma^{2} \Delta t} \int_{-t_{r}}^{t_{r}} \cos ^{2}\left(2 \pi f_{0} t+\phi_{n}\right) d t \\
& =\frac{V_{0}^{2}}{\sigma^{2} \Delta t}\left(t_{r}+\frac{\cos \left(2 \phi_{n}\right) \sin \left(4 \pi f_{0} t_{r}\right)}{4 \pi f}\right) \\
& =\frac{V_{0}^{2}}{2 \sigma^{2} \Delta t f_{0}},
\end{aligned}
$$

where the last step assumes $t_{r}$ has been chosen at the optimal value of $1 / 2 f_{0}$, and without loss of generality the zero of $t$ has been chosen at the center of the fit interval.

Note that choosing to fit the function in windows of width $2 t_{r}=1 / f_{0}$ has resulted in an expression for $\alpha$ that is independent of the fitted parameter $\phi_{n}$. Indeed, the integral in Eq. (B7) is independent of $\phi_{n}$ for all integration regions of width $2 t_{r}=1 / f_{0}$, regardless of where they are centered. Thus, knowledge of the membrane frequency $f_{0}$ implies that the standard error in the fitted parameter $\phi_{n}$ is only related to the physical parameters set for the experiment. Also note that on converting the sum to an integral, we would expect the expressions to be approximately equal. Observe that the right-Riemann sum $\alpha \Delta t$ would overestimate the integral of any monotonically increasing function in the interval, while underestimating for a monotonically decreasing function. If the parameter is fitted such that it falls roughly within the center of the window each time (i.e., we place the window roughly where we expect the crossing), the effects of overestimating and underestimating the symmetric function under the integral will roughly balance out. 
To obtain the standard deviation $\Delta \phi_{n}$, we should take $\Delta \chi^{2}=1$ in Eq. (B5), giving

$$
\Delta \phi=\alpha^{-1 / 2} .
$$

With this, the accuracy in the Johnson-noise model is

$$
\begin{aligned}
N_{J} & =4 \pi^{2} \alpha \\
& =\frac{2 \pi^{2} V_{0}^{2}}{\sigma^{2} \Delta t f_{0}} .
\end{aligned}
$$

The per-point standard deviation depends on the measurement bandwidth of the amplifier and on the system noise. In the best case, it will be set by the Johnson noise of the cold resistor [41], giving

$$
\sigma=\sqrt{4 k_{B} T_{c} R_{0} B}
$$

where $B$ is the measurement bandwidth (defined using the single-sided frequency convention), and the factor 4 appears because the bandpass filter presents on open load except near resonance. In order that successive points are independent but no data are lost, the sampling interval should be related to the bandwidth by $B=1 / 2 \Delta t$. Thus,

$$
\sigma^{2} \Delta t=2 k_{B} T_{c} R_{0} .
$$

Substituting into Eq. (B11) gives for the phase uncertainty in the interval which we chose to fit

$$
\delta \phi_{n}=\frac{\sqrt{4 f_{0} k_{B} T_{c} R_{0}}}{V_{0}} .
$$

Over many oscillations, $V_{0}$ fluctuates, but its root-meansquare value is $V_{S}$, given by Eq. (B2). Substituting this and Eq. (B15) into Eq. (B13) gives us a model for the accuracy of the clock:

$$
\begin{aligned}
N_{J} & =\frac{2 \pi^{2}}{f_{0} k_{B} T_{c} R_{0}}\left\langle V_{S}^{2}\right\rangle \\
& =\frac{2 \pi^{2}}{Q_{f}} \frac{T_{h}+T_{c}}{T_{c}} .
\end{aligned}
$$

The clock creates entropy because the power carried by the electrical oscillation is converted to heat in the cold resistor. The entropy creation rate can be written as

$$
\dot{S}=k_{B} \frac{T_{h}-T_{c}}{T_{c}} \frac{f_{0}}{Q_{f}}
$$

since the net power transferred is $P_{0}=k_{B}\left(T_{h}-T_{c}\right) f_{0} / Q_{f}$. Combining this expression with Eq. (B18) gives the accuracy in terms of the entropy created:

$$
\begin{aligned}
N_{J} & =2 \pi^{2} \frac{T_{h}+T_{c}}{T_{h}-T_{c}} \frac{\Delta S_{\text {tick }}}{k_{B}} \\
& \approx 2 \pi^{2} \frac{\Delta S_{\text {tick }}}{k_{B}}, \quad \text { for } T_{h} \gg T_{c} .
\end{aligned}
$$

where $\Delta S_{\text {tick }} \equiv \dot{S} / f_{0}$ is the entropy generated per tick. This best case scenario (i.e., smallest $\sigma$ ) provides an upper bound for the best achievable accuracy of an experiment of this type. Thus, we can expect this model to overestimate the accuracy compared to that coming from a live experiment. Similar expressions to Eq. (B21) hold for a classical clock defined by transitions on a network [26] and for an autonomous quantum clock [4]; however, in both these cases the factor $2 \pi^{2}$ is replaced by $1 / 2$.

\section{Measuring time from an optomechanical signal}

In this section we proceed to build a classical model that predicts the accuracy, which we call $N_{C}$, for a scheme that is more fitting to our experimental setup. Figure 6 shows the optomechanical setup, which serves as the clock of our experiment. The clock works by illuminating a tank circuit containing a vibrating membrane with a rf tone of power $P_{c}$ [Fig. 6(a)]. The thermal motion of the membrane modulates the phase of the reflected signal, and from this signal the ticks are derived. This is the principle of the clock realized in our experiment. The advantage of this clock over the version of Fig. 5 is that the reflected signal can be increased by increasing $P_{c}$ as well as by heating the membrane more strongly. As we show in this section, this clock obeys a similar entropyaccuracy relation to Eq. (B21). The voltage incident on the tank circuit is

$$
V_{\text {in }}(t)=V_{c} \cos \left(2 \pi f_{c} t\right),
$$

where $V_{c}=\sqrt{2 R_{0} P_{c}}$ and $f_{c}$ are, respectively, the amplitude and frequency of the illumination signal, and the characteristic impedance of the transmission line is assumed equal to $R_{0}$. The reflected amplitude is therefore

$\sqrt{b^{2}-4 \times a \times c} V_{\text {out }}(t)=\Gamma V_{c} \cos \left(2 \pi f_{c} t+\beta x(t)\right)$,

where $\Gamma$ is the cavity reflection coefficient, $\beta$ is the mechanical coupling strength, and $x(t)$ is the instantaneous membrane displacement. The phase reference plane is assumed to be chosen so that the phase is zero at the membrane's equilibrium position. The membrane vibrates with a mechanical temperature $T_{h}$. If its quality factor is high, the mechanical amplitude $x_{0}$ and phase $\phi$ are approximately constant over one oscillation cycle, meaning that the displacement is

$$
x(t)=x_{0} \cos \left(2 \pi f_{0} t+\phi\right) .
$$


(a)

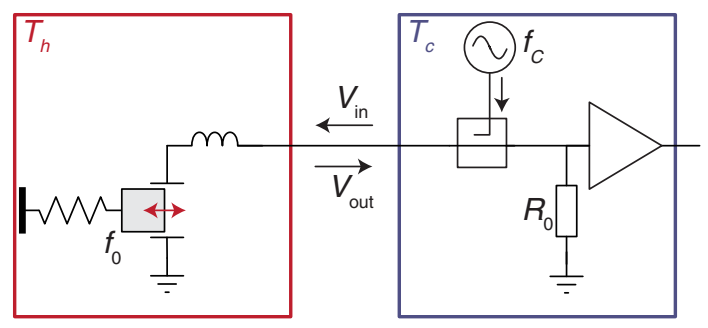

(b)

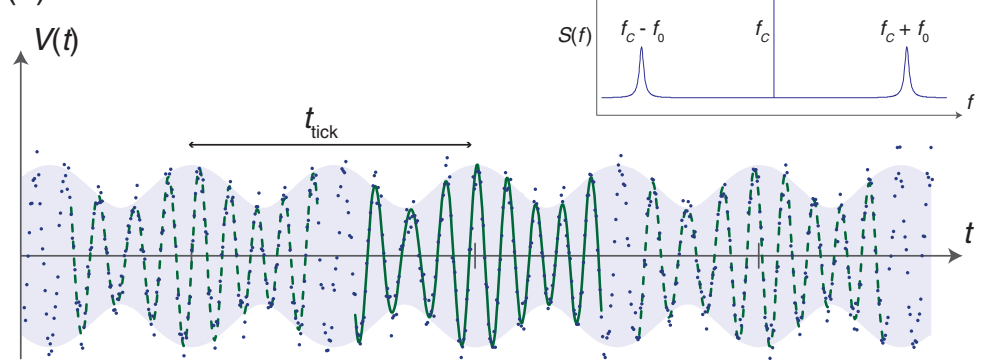

FIG. 6. The thermomechanical clock. (a) The setup. An optomechanical circuit consists of an $L C$ tank circuit whose capacitance, and therefore frequency, is modulated by a thermomechanical resonator at temperature $T_{h}$. To use the vibrations in a clock, the tank circuit is illuminated by a carrier tone $V_{\text {in }}$ at frequency $f_{c}$, giving rise to a reflected signal $V_{\text {out }}$ which is passed to a cold matched resistor and amplifier. The effect of the vibrations is to modulate the phase of $V_{\text {out }}$. (b) Sketch of the resulting voltage record at the amplifier input (points), with fits (lines) from which each tick is extracted. The modulation envelope is indicated by the shaded background. Inset: power spectrum at the amplifier input, showing uniform noise background, central delta-function peak from the carrier, and two thermomechanical sidebands. In the experiment, a demodulation circuit was applied after the amplifier [as in Fig. 2(d)] because it makes ticks practically easier to identify in the record. However, the demodulator cannot improve the clock accuracy because it cannot increase the timing information present in the signal $V(t)$; in fact, a detailed calculation would show that the accuracy is unchanged. For simplicity, the demodulator is therefore omitted from this model.

In this experiment, the electromechanical coupling is weak, meaning that $\beta x_{0} \ll 1$. This means that we can substitute Eq. (B24) into Eq. (B23) and expand to lowest order in $\beta x_{0}$, giving

$$
V_{\text {out }}(t)=\Gamma V_{c}\left(\cos \left(2 \pi f_{c} t\right)-\beta x_{0} \sin \left(2 \pi f_{c} t\right) \cos \left(2 \pi f_{0} t+\phi\right)\right) .
$$

In other words, the reflected signal is modulated at frequency $f_{0}$ with phase $\phi$, as sketched in Fig. 6(b). Each full cycle of the modulation is one period of the clock. To generate ticks, the clock must identify a particular point of the modulation cycles, which implies it must precisely identify $\phi$. As in Appendix B 1, we want to know how accurately this can be done in principle. Again, we imagine we have obtained a set of experimental data and wish to know how accurately the $n$th tick can be identified. We proceed by fitting the function,

$$
V\left(t \mid \phi_{n}\right)=A_{0} \cos \left(2 \pi f_{c} t\right)+A_{1} \sin \left(2 \pi f_{c} t\right) \cos \left(2 \pi f_{0} t+\phi_{n}\right),
$$

in windows of width $1 / f_{0}$ around the expected tick locations. The parameters $A_{0}, A_{1}, f_{c}$, and $f_{0}$ can be extracted over several recent cycles, and are thus known values. Therefore, just as in Appendix B 1, we are performing a one-parameter fit.

We imagine that for some dataset we minimize Eq. (B4) for the function in Eq. (B26), which gives us the optimal parameter $\phi_{n}^{*}$. We now want to know, what is the error in this fit given the optomechanical setup we have described? We follow the recipe give in the previous section and proceed to calculate the curvature parameter of our model:

$$
\begin{aligned}
\alpha= & \frac{1}{\sigma^{2} \Delta t} \int_{-t_{r}}^{t_{r}}\left(\frac{\partial V\left(t \mid \phi_{n}\right)}{\partial \phi_{n}}\right)^{2} d t \\
= & \frac{A_{1}^{2}}{\sigma^{2} \Delta t} \int_{-t_{r}}^{t_{r}} \sin ^{2}\left(2 \pi f_{c} t\right) \sin ^{2}\left(2 \pi f_{0} t+\phi_{n}\right) d t \\
= & \frac{A_{1}^{2}}{4 \sigma^{2} \Delta t} \int_{-t_{r}}^{t_{r}} 1-\cos \left(4 \pi f_{0} t+2 \phi_{n}\right)-\cos \left(4 \pi f_{c} t\right) \\
& -\frac{\cos \left(4 \pi\left(f_{c}+f_{0}\right) t+2 \phi_{n}\right)}{2} \\
& +\frac{\cos \left(4 \pi\left(f_{c}-f_{0}\right) t-2 \phi_{n}\right)}{2} d t
\end{aligned}
$$

where $t_{r}=1 / 2 f_{0}$ is the fit range. Since the fit window extends over many cycles of the carrier tone, i.e., $t_{r} \gg 1 / f_{c}$, the last three oscillatory terms make a negligible contribution to the integral, leaving

$$
\begin{aligned}
\alpha & =\frac{A_{1}^{2}}{4 \sigma^{2} \Delta t} \int_{-t_{r}}^{t_{r}} 1-\cos \left(4 \pi f_{0} t+2 \phi_{n}\right) d t \\
& =\frac{A_{1}^{2}}{2 \sigma^{2} \Delta t}\left(t_{r}-\frac{\cos 2 \phi_{n}}{4 \pi f_{0}} \sin \left(4 \pi f_{0} t_{r}\right)\right) \\
& =\frac{A_{1}^{2}}{4 f_{0} \sigma^{2} \Delta t} .
\end{aligned}
$$

Since the tank circuit presents an open electrical impedance except at its resonance frequency, the Johnson noise again obeys Eq. (B15), leading to

$$
\alpha=\frac{A_{1}^{2}}{8 f_{0} k_{B} T_{c} R_{0}},
$$


which implies that $\delta \phi_{n}=\sqrt{8 f_{0} k_{B} T_{c} R_{0}} / A_{1}$ and

$$
N_{C}=4 \pi^{2} \alpha=\frac{\pi^{2}\left\langle A_{1}^{2}\right\rangle}{2 f_{0} k_{B} T_{c} R_{0}} .
$$

To connect this to thermodynamic quantities in the experiment, we recognize that $A_{1}$ is related to the combined power $P_{\mathrm{SB}}$ in the two sidebands by

$$
P_{\mathrm{SB}}=\frac{\left\langle A_{1}^{2}\right\rangle}{4 R_{0}} .
$$

Entropy is created because the reflection from the tank circuit containing the hot resonator leads to irreversible heating in the cold resistor. Equation (B25) and the inset of Fig. 6(b) show that there are potentially two contributions to the heat: the reflected carrier, which is a coherent monochromatic tone at frequency $f_{c}$, and the two incoherent sidebands centered at $f_{c} \pm f_{0}$. However, the carrier contains no information about $x(t)$. In principle (although this was not implemented in our experiment) a narrow band filter could be used to direct this portion of the spectrum back toward the tank circuit without affecting the accuracy of the clock. Thus the reflected carrier does not contribute to the fundamental entropy cost of the clock. Instead, the unavoidable entropy increase is determined by the two sidebands, which dissipate heat $P_{\mathrm{SB}}$ in the cold resistor. The entropy creation rate is

$$
\dot{S}=\frac{P_{\mathrm{SB}}}{T_{c}} .
$$

In contrast to Eq. (B19), there is no decrease of entropy in the hot element, because illuminating the membrane at the cavity frequency does not cool it. Thus we can reexpress Eq. (B34) in terms of the entropy generated per tick, leading to

$$
N_{C}=\frac{2 \pi^{2}}{k_{B}} \Delta S_{\text {tick }}
$$

where

$$
\Delta S_{\text {tick }}=\frac{P_{\mathrm{SB}}}{f_{0} T_{c}} .
$$

Equation (B37) is the fundamental entropy-accuracy relation for the optomechanical clock.

There is one more adjustment which must be made to compare Eqs. (B37) and (B38) to experiment. The derivation above assumed that the amplifier noise is much less than the Johnson noise of the cold resistor. Although this is perfectly possible, it is also common (and is the case in our experiment) that other noise sources contribute, leading to a decrease in accuracy that reflects technical imperfections in the voltage measurement rather than any fundamental bound. To account for this possibility, Eq. (B37) should be generalized to

$$
N_{C}=\frac{2 \pi^{2}}{k_{B}} \frac{T_{c}}{T_{N}} \Delta S_{\text {tick }}
$$

where $T_{N}$ is the effective temperature, including the Johnson noise of the cold resistor, determined by the noise in the record.

To evaluate Eq. (B39) from the experiment, we express its components in terms of the output signal's power spectrum $\mathcal{S}_{V V}$, which is proportional to the modulus squared of the Fourier transform of the record $V(t)$. In this language, the effective temperature is given by

$$
T_{N}=\frac{\mathcal{S}_{V V}^{(N)}}{4 k_{B} R_{0}} .
$$

Here $\mathcal{S}_{V V}^{(N)}$ is the single-sided average spectral density of the noise in the Fourier transformed signal, i.e., the average background level of the power spectrum. In terms of the power spectrum, the heat $P_{\mathrm{SB}}$ in the cold resistor is given by integrating the excess spectral density (i.e., the signal) above the noise background, the integral running over both sidebands, $\int \mathcal{S}_{V V}^{(S)}(f) d f$. Thus the classical model predicts the accuracy from the experimental data to be

$$
\begin{aligned}
N_{C} & =\frac{2 \pi^{2}}{f_{0}} \frac{A_{1}^{2}}{\mathcal{S}_{V V}^{(N)}} \\
& =\frac{8 \pi^{2}}{f_{0}} \frac{P_{\mathrm{SB}} R_{0}}{\mathcal{S}_{V V}^{(N)}} \\
& =\frac{8 \pi^{2}}{f_{0}} \frac{\int \mathcal{S}_{V V}^{(S)}(f) d f}{\mathcal{S}_{V V}^{(N)}}
\end{aligned}
$$

where the second line follows from Parseval's theorem. In practice, our analysis applies Eq. (B43) to the record of the demodulated voltage as in Fig. 2(d). Since demodulation does not change the signal-to-noise ratio, Eq. (B43) remains valid, with the integral now taken over the single signal peak.

\section{APPENDIX C: ANALYTIC PREDICTION FOR THE THERMODYNAMIC PRECISIONS}

Here, we derive an analytic prediction for the thermodynamic precision in our experimental setup. This prediction is then used to fit the curves in Figs. 4 and 7. Recall from Eq. (4) that the "precision" in the sense of a thermodynamic uncertainty relation (TUR) is a statistical measure that characterizes the fluctuations of an observable $F$ and is defined as 


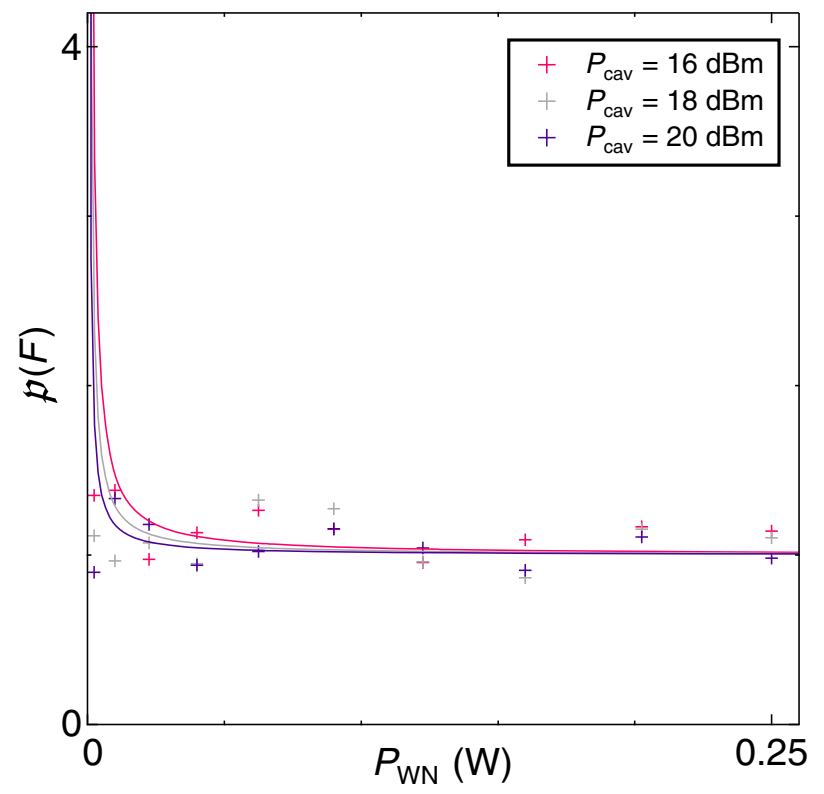

FIG. 7. Precision $\mathfrak{p}(F)$ of the demodulated signal as a function of mechanical drive power $P_{\mathrm{WN}}$ for different settings of the cavity illumination power $P_{\text {cav }}$ in the range 16-20 dBm. Crosses show precision calculated from the voltage record data using Eqs. (4) and (7). Curves show predictions from Eq. (C31). The free parameter $\alpha$ is set by fitting to the data with $P_{\text {cav }}=8 \mathrm{dBm}$, then held fixed to generate the other curves.

$$
\mathfrak{p}(F) \equiv \frac{|\langle F\rangle|^{2}}{\operatorname{Var} F}
$$

where $\langle\cdots\rangle$ denotes the expectation value. To confirm that the precision computed from experimental data in Figs. 4 and 7 has the expected form, we also calculate it analytically, beginning with the predicted output voltage from Eq (B25). The voltage record $V(t)$ is generated from the received voltage $V_{\text {out }}(t)$ by the demodulation circuit in Fig. 2(d). The first effect of this circuit is to multiply the received voltage by $\sin 2 \pi f_{c} t$, thus generating a signal:

$$
\begin{aligned}
V_{\text {mixed }}(t) & =V_{\text {out }}(t) \sin 2 \pi f_{c} t \\
& =\Gamma V_{c}\left(\cos 2 \pi f_{c} t \sin 2 \pi f_{c} t-\beta x(t) \sin ^{2} 2 \pi f_{c} t\right) .
\end{aligned}
$$

The second effect is to bandpass filter (BPF) this signal around the mechanical frequency $f_{0}$ to generate the demodulated voltage,

$$
\begin{aligned}
V(t) & =\operatorname{BPF}\left\{V_{\text {mixed }}(t)\right\} \\
& =-\Gamma V_{c} \beta x(t),
\end{aligned}
$$

which as expected is proportional to the instantaneous displacement. This is the voltage that would be recorded if there were no noise in the measurement circuit. Since noise is inevitably present, we include it by replacing Eq. (C5) with

$$
V(t)=-\Gamma V_{c} \beta x(t)+\xi(t),
$$

where $\xi(t)$ is the measurement noise voltage.

We can now calculate the precision according to Eq. (C1). The observable according to Eq. (7) is

$$
F \equiv\left[V^{2}(t)\right],
$$

where $[\cdots]$ denotes a time average. Substituting from Eq. (C6) gives:

$$
\begin{aligned}
\langle F\rangle & =\left\langle\left[\left(-\Gamma V_{c} \beta x(t)+\xi(t)\right)^{2}\right]\right\rangle \\
& =\Gamma^{2} V_{c}^{2} \beta^{2}\left\langle\left[x^{2}(t)\right]\right\rangle+\left\langle\left[\xi^{2}(t)\right]\right\rangle \\
& =\Gamma^{2} V_{c}^{2} \beta^{2}\left\langle x^{2}(t)\right\rangle+\left\langle\xi^{2}(t)\right\rangle \\
& =\Gamma^{2} V_{c}^{2} \beta^{2} X^{2}+\Xi^{2} .
\end{aligned}
$$

Here Eq. (C9) follows because the displacement and the measurement noise are uncorrelated, Eq. (C10) because the order of time average and expectation value can be exchanged, and Eq. (C11) by defining $X$ and $\Xi$ to be the root-mean-square amplitudes of displacement and of measurement noise, respectively.

To calculate the variance, we also need

$$
\begin{aligned}
\left\langle F^{2}\right\rangle= & \left\langle\left[V^{2}(t)\right]^{2}\right\rangle \\
= & \left\langle\left[\left(-\Gamma V_{c} \beta x(t)+\xi(t)\right)^{2}\right]^{2}\right\rangle \\
= & \Gamma^{4} V_{c}^{4} \beta^{4}\left\langle\left[x^{2}(t)\right]^{2}\right\rangle+2 \Gamma^{2} V_{c}^{2} \beta^{2}\left\langle\left[x^{2}(t)\right]\left[\xi^{2}(t)\right]\right\rangle \\
& +\left\langle\left[\xi^{2}(t)\right]^{2}\right\rangle \\
& \\
= & \Gamma^{4} V_{c}^{4} \beta^{4}\left\langle\left[x^{2}(t)\right]^{2}\right\rangle+2 \Gamma^{2} V_{c}^{2} \beta^{2} X^{2} \Xi^{2}+\Xi^{4} .
\end{aligned}
$$

Here Eq. (C14) follows by expanding the bracket and using that $[x(t)]=0$ for integration over an integer number of cycles, and Eq. (C15) uses that $x(t)$ and $\xi(t)$ are uncorrelated and that the correlation time of $\xi(t)$ is much shorter than the averaging interval.

To evaluate the first term in Eq. (C15), we separate $x(t)$ into its two quadratures by writing

$$
x(t)=x_{1} \cos 2 \pi f_{0} t+x_{2} \sin 2 \pi f_{0} t .
$$

Provided the averaging window is shorter than the mechanical damping time, which is well satisfied in Fig. 4, then $x_{1}$ and $x_{2}$ can be taken as constant within each average, leading to 
(a)

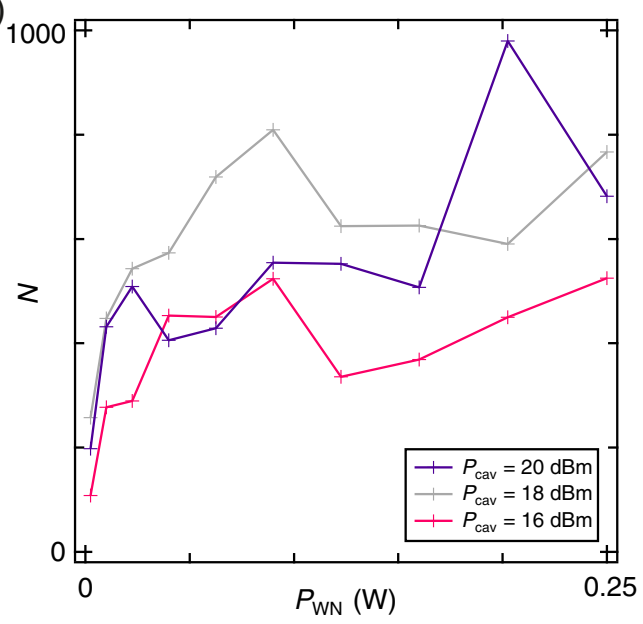

(b)

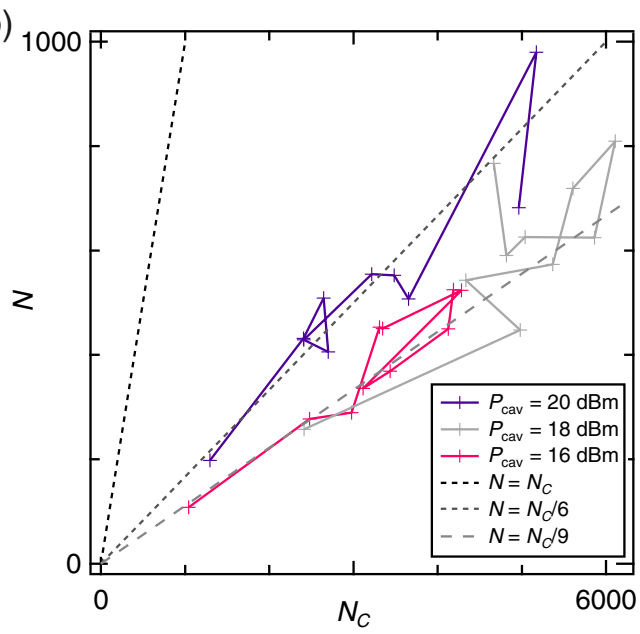

FIG. 8. (a) Accuracy $N$ of the clock versus the white-noise power $P_{\mathrm{WN}}$ for cavity drive powers in the range $16-20 \mathrm{dBm}$. (b) Accuracy $N$ of the clock versus $N_{C}$ for cavity drive powers in the range $16-20 \mathrm{dBm}$. The black dotted line is a guide for the eye to show the expected gradient should the extracted accuracy and the theoretical accuracy predicted from the entropy production be equal. The dark gray dotted line shows the approximate gradient of the $20 \mathrm{dBm}$ data of $N=N_{C} / 6$ and the light gray line shows the approximate gradient of the 16 and $18 \mathrm{dBm}$ data of $N=N_{C} / 9$.

$$
\begin{aligned}
\left\langle\left[x^{2}(t)\right]^{2}\right\rangle= & \left\langle\left[\left(x_{1} \cos ^{2} 2 \pi f_{0} t+x_{2} \sin 2 \pi f_{0} t\right)^{2}\right]^{2}\right\rangle \\
= & \left\langle\left[ x_{1}^{2} \cos ^{2} 2 \pi f_{0} t+2 x_{1} x_{2} \cos 2 \pi f_{0} t \sin 2 \pi f_{0} t\right.\right. \\
& \left.\left.+x_{2}^{2} \sin ^{2} 2 \pi f_{0} t\right]^{2}\right\rangle \\
= & \left\langle\left(\left(x_{1}^{2}+x_{2}^{2}\right) / 2\right)^{2}\right\rangle \\
= & \frac{1}{4}\left\langle x_{1}^{4}+2 x_{1}^{2} x_{2}^{2}+x_{2}^{4}\right\rangle \\
= & \frac{1}{2}\left(\left\langle x_{1}^{4}\right\rangle+\left\langle x_{1}^{2}\right\rangle^{2}\right)
\end{aligned}
$$

Equation (C19) follows because $\left[\cos ^{2} 2 \pi f_{0} t\right]=\left[\sin ^{2} 2 \pi f_{0} t\right]=$ $\frac{1}{2}$ and $\left[\cos 2 \pi f_{0} t \sin 2 \pi f_{0} t\right]=0$. Equation (C21) follows because the statistics of $x_{1}$ and $x_{2}$ are equivalent.

To evaluate Eq. (C21), we use the fact that $x_{1}$ is a response to many random impulses received by the resonator at different times. It therefore follows a normal distribution, which implies that

$$
\left\langle x_{1}^{4}\right\rangle=3\left\langle x_{1}^{2}\right\rangle^{2}
$$

and therefore

$$
\begin{aligned}
\left\langle\left[x^{2}(t)\right]^{2}\right\rangle & =2\left\langle x_{1}^{2}\right\rangle^{2} \\
& =2 X^{4} .
\end{aligned}
$$

Substituting Eq. (C24) into Eq. (C15) leads to

$$
\left\langle F^{2}\right\rangle=\Gamma^{4} V_{c}^{4} \beta^{4} X^{4}+\left(\Gamma^{2} V_{c}^{2} \beta^{2} X^{2}+\Xi^{2}\right)^{2} .
$$

The precision according to Eq. (C1) is therefore

$$
\begin{aligned}
\mathfrak{p}(F) & =\frac{\langle F\rangle^{2}}{\left\langle F^{2}\right\rangle-\langle F\rangle^{2}} \\
& =\frac{\left(\Gamma^{2} V_{c}^{2} \beta^{2} X^{2}+\Xi^{2}\right)^{2}}{\Gamma^{4} V_{c}^{4} \beta^{4} X^{4}} \\
& =\left(1+\frac{\Xi^{2}}{\Gamma^{2} V_{c}^{2} \beta^{2} X^{2}}\right)^{2} .
\end{aligned}
$$

Let us now examine the limiting cases of the precision with this choice of $F$. In the first case, when the noise is small compared to the signal, we have

$$
\lim _{\Gamma V_{c} \beta X \gg \Xi} \mathfrak{p}(F) \rightarrow 1,
$$

while, on the other hand, when the noise dominates the signal, we find

$$
\lim _{\Xi \gg \Gamma V_{c} \beta X} \mathfrak{p}(F) \rightarrow \infty
$$

The reason that this model predicts an apparently infinite precision is that white noise contains independent contributions to $F$ at infinitely many frequencies. By contrast, Eq. (C29) describes a situation where the clock output is dominated by the mechanical signal, which exists in a narrow frequency range but whose amplitude is subject to long-lived fluctuations. In fact, the limit of Eq. (C30) is unphysical because the power needed to amplify the entire spectrum of white noise would be infinite.

To relate Eq. (C28) to experimental parameters, we use that $V_{c}^{2}$ is proportional to the cavity illumination power 

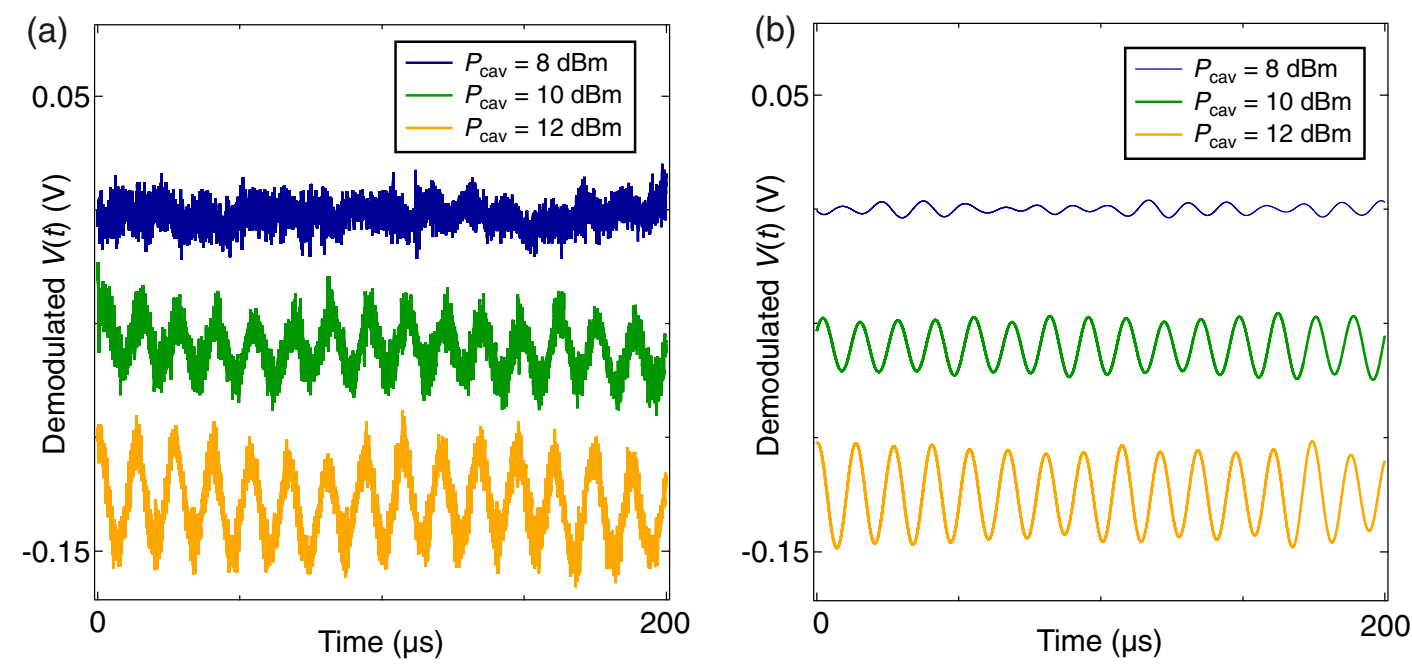

FIG. 9. The demodulated cavity output signal before (a) and after (b) filtering as a function of time for input powers of 8, 10, and $12 \mathrm{dBm}$ to the cavity with $0.25 \mathrm{~W}$ white-noise input from port 3 . The traces are vertically offset for clarity.

$P_{\text {cav }}$ and that $X^{2}$ is proportional to the mechanical drive power $P_{\mathrm{WN}}$. Then the expected precision is

$$
\mathfrak{p}(F)=\left(1+\frac{\alpha}{P_{\mathrm{cav}} P_{\mathrm{WN}}}\right)^{2},
$$

where $\alpha$ is a constant proportional to the intensity of the measurement noise. Equation (C31) is the fitting function used in Fig. 4, with $\alpha$ as the fit parameter. To plot the curves in Fig. 4, we fit the data for $P_{\text {cav }}=8 \mathrm{dBm}$ to extract the value $\alpha=8.6 \times 10^{-5} \mathrm{~W}^{2}$. We then use this value of $\alpha$ to predict $\mathfrak{p}(F)$ for all the other datasets in the figure, with no free parameters. There is moderately good agreement with all datasets, with discrepancies being consistent with an excess of measurement noise at high illumination power;

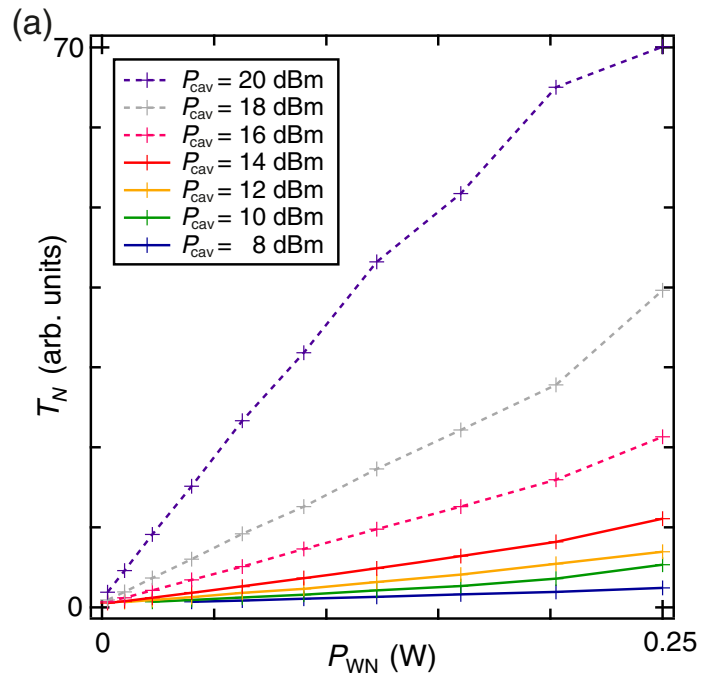

importantly, the limits derived in Eqs. (C29) and (C30) are observed in our experimental data. This agreement confirms that our physical model and numerical analysis are sound.

\section{APPENDIX D: OVERDRIVING THE MEMBRANE}

Above $14 \mathrm{dBm}$ we enter the nonlinear regime of the membrane's motion. As can be seen from Fig. 8(a), for these higher drive powers the relationship between accuracy $N$ and white-noise power $P_{\mathrm{WN}}$ is more erratic. The general trend of accuracy increasing with $P_{\mathrm{WN}}$ is still there for the lower values of $P_{\mathrm{WN}}$, but then the accuracy saturates and unstable dynamics dominates the motion of the membrane. As discussed in the main text, the saturation

(b)

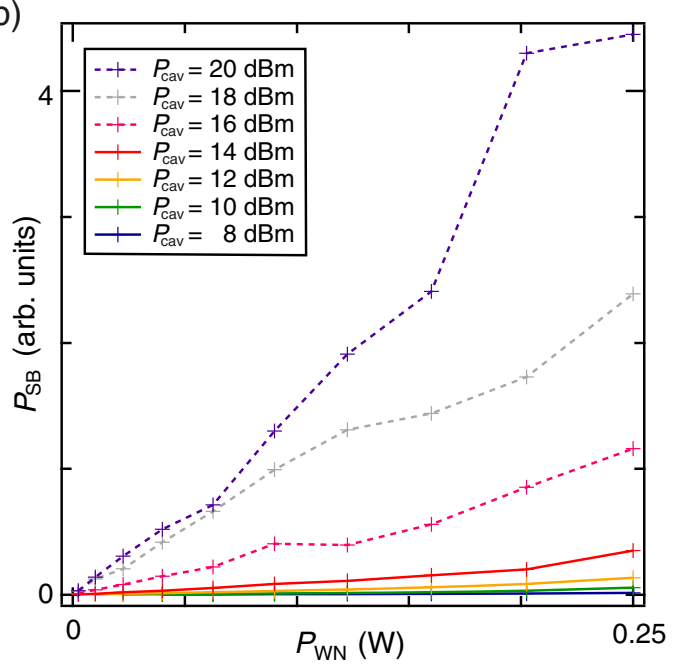

FIG. 10. (a) Noise temperature of the system $T_{N}$ increasing as the cavity drive power and white-noise power increase for cavity drive powers in the range $8-20 \mathrm{dBm}$. (b) The increase in the power of the sideband with increase in the white-noise power for different cavity drive powers in the range $8-20 \mathrm{dBm}$. For both (a) and (b) the higher drive powers have dotted lines and the data shown in the main text have full lines. 
of accuracy is to be expected. Surprisingly, Fig. 8(b) shows that the higher power measurements show better agreement with the theoretical predictions of Eq. (3) [or equally Eq. (B39)] with a gradient of $1 / 9$ for the 16 and $18 \mathrm{dBm}$ datasets and $1 / 6$ for the $20 \mathrm{dBm}$ dataset.

\section{APPENDIX E: MEASUREMENTS AT LOW CAVITY ILLUMINATION POWER}

Figure 3 shows only measurements down to a drive power of $P_{\text {cav }}=8 \mathrm{dBm}$. This is due to the fact that below this drive power the signal is weak and identifying the oscillations becomes difficult. Example traces corresponding to a whitenoise power of $0.25 \mathrm{~W}$ with 8,10 , and $12 \mathrm{dBm}$ drive powers are shown in Fig. 9 before [Fig. 9(a)] and after [Fig. 9(b)] filtering. As can be seen in the signal for a 12 and $10 \mathrm{dBm}$ drive oscillations can easily be identified; however, they are much fainter in the $8 \mathrm{dBm}$ signal.

\section{APPENDIX F: SYSTEM NOISE TEMPERATURE}

As can be seen from Fig. 10(a), the noise temperature of the system increases for both an increased cavity drive or an increased white-noise power, $P_{\mathrm{WN}}$. Figure 10(b) shows that the power in the sideband, used in the entropy calculations [Eq. (B43)], increases approximately linearly with $P_{\mathrm{WN}}$.

[1] T. Nicholson et al., Systematic Evaluation of an Atomic Clock at $2 \times 10^{-18}$ Total Uncertainty, Nat. Commun. 6 , 6896 (2015).

[2] B. Odom, D. Hanneke, B. D'Urso, and G. Gabrielse, New Measurement of the Electron Magnetic Moment Using a One-Electron Quantum Cyclotron, Phys. Rev. Lett. 97, 030801 (2006).

[3] V. Rajaraman, IEEE Standard for Floating Point Numbers, Resonance 21, 11 (2016).

[4] P. Erker, M. T. Mitchison, R. Silva, M. P. Woods, N. Brunner, and M. Huber, Autonomous Quantum Clocks: Does Thermodynamics Limit Our Ability to Measure Time?, Phys. Rev. X 7, 031022 (2017).

[5] P. Erker, The Quantum Hourglass, Master's thesis, ETH Zurich, 2014.

[6] A. C. Barato and U. Seifert, Cost and Precision of Brownian Clocks, Phys. Rev. X 6, 041053 (2016).

[7] G. J. Milburn and T. J. Milburn, A Quantum Optomechanical Mach Clock, arXiv:1708.02369.

[8] G. J. Milburn, The Thermodynamics of Clocks, Contemp. Phys. 61, 69 (2020).

[9] E. Schwarzhans, M. P. E. Lock, P. Erker, N. Friis, and M. Huber, Autonomous Temporal Probability Concentration: Clockworks and the Second Law of Thermodynamics, Phys. Rev. X 11, 011046 (2021).

[10] J. Goold, M. Huber, A. Riera, L. del Rio, and P. Skrzypczyk, The Role of Quantum Information in Thermodynamics-A Topical Review, J. Phys. A 49, 143001 (2016).
[11] N. Y. Halpern and D. T. Limmer, Fundamental Limitations on Photoisomerization from Thermodynamic Resource Theories, Phys. Rev. A 101, 042116 (2020).

[12] G. Manzano, R. Silva, and J. M. R. Parrondo, Autonomous Thermal Machine for Amplification and Control of Energetic Coherence, Phys. Rev. E 99, 042135 (2019).

[13] O. Abah, R. Puebla, A. Kiely, G. De Chiara, M. Paternostro, and S. Campbell, Energetic Cost of Quantum Control Protocols, New J. Phys. 21, 103048 (2019).

[14] F. Clivaz, R. Silva, G. Haack, J. B. Brask, N. Brunner, and M. Huber, Unifying Paradigms of Quantum Refrigeration: A Universal and Attainable Bound on Cooling, Phys. Rev. Lett. 123, 170605 (2019).

[15] A. S. L. Malabarba, A. J. Short, and P. Kammerlander, Clock-Driven Quantum Thermal Engines, New J. Phys. 17, 045027 (2015).

[16] M. P. Woods, R. Silva, and J. Oppenheim, Autonomous Quantum Machines and Finite-Sized Clocks, Ann. Henri Poincaré 20, 125 (2019).

[17] C. Gogolin and J. Eisert, Equilibration, Thermalisation, and the Emergence of Statistical Mechanics in Closed Quantum Systems, Rep. Prog. Phys. 79, 056001 (2016).

[18] J. Eisert, M. Friesdorf, and C. Gogolin, Quantum ManyBody Systems Out of Equilibrium, Nat. Phys. 11, 124 (2015).

[19] M. T. Mitchison, Quantum Thermal Absorption Machines: Refrigerators, Engines and Clocks, Contemp. Phys. 60, 164 (2019).

[20] I. A. Martínez, É. Roldán, L. Dinis, D. Petrov, J. M. R. Parrondo, and R. A. Rica, Brownian Carnot Engine, Nat. Phys. 12, 67 (2016).

[21] J. Roßnagel, S. T. Dawkins, K. N. Tolazzi, O. Abah, E. Lutz, F. Schmidt-Kaler, and K. Singer, A Single-Atom Heat Engine, Science 352, 325 (2016).

[22] J. P. Garrahan, Simple Bounds on Fluctuations and Uncertainty Relations for First-Passage Times of Counting Observables, Phys. Rev. E 95, 032134 (2017).

[23] K. Proesmans and C. Van den Broeck, Discrete-Time Thermodynamic Uncertainty Relation, Europhys. Lett. 119, 20001 (2017).

[24] A. C. Barato, R. Chetrite, A. Faggionato, and D. Gabrielli, Bounds on Current Fluctuations in Periodically Driven Systems, New J. Phys. 20, 103023 (2018).

[25] G. Falasco, M. Esposito, and J.-C. Delvenne, Unifying Thermodynamic Uncertainty Relations, New J. Phys. 22, 053046 (2020).

[26] A. C. Barato and U. Seifert, Thermodynamic Uncertainty Relation for Biomolecular Processes, Phys. Rev. Lett. 114, 158101 (2015).

[27] White noise is a high-entropy signal and, while it is not straightforward to assign a temperature to it, we do not require a low-entropy source of work to prepare it (one can expect that it is reasonably abundant in out-of-equilibrium environments). The notion of temperature itself often becomes ill defined in microscopic contexts, as deviations from thermal equilibrium are more frequent and noticeable, and selective coupling to certain frequencies can yield multiple notions of temperature even for an ideal blackbody.

[28] K. W. Lehnert, Introduction to Microwave Cavity Optomechanics, in Cavity Optomechanics: Nano- and Microme- 
chanical Resonators Interacting with Light, edited by M. Aspelmeyer, T. Kippenberg, and F. Marquardt (Springer, Berlin, 2014), pp. 233-252.

[29] T. Bagci et al., Optical Detection of Radio Waves through a Nanomechanical Transducer, Nature (London) 507, 81 (2014).

[30] N. Ares, T. Pei, A. Mavalankar, M. Mergenthaler, J. H. Warner, G. A. D. Briggs, and E. A. Laird, Resonant Optomechanics with a Vibrating Carbon Nanotube and a RadioFrequency Cavity, Phys. Rev. Lett. 117, 170801 (2016).

[31] K. R. Brown, J. Britton, R. J. Epstein, J. Chiaverini, D. Leibfried, and D. J. Wineland, Passive Cooling of a Micromechanical Oscillator with a Resonant Electric Circuit, Phys. Rev. Lett. 99, 137205 (2007).

[32] T. Faust, P. Krenna, S. Manus, J. Kotthaus, and E. Weig, Microwave Cavity-Enhanced Transduction for Plug and Play Nanomechanics at Room Temperature, Nat. Commun. 3, 728 (2012).

[33] A. N. Pearson, K. E. Khosla, M. Mergenthaler, G. A. D. Briggs, E. A. Laird, and N. Ares, Radio-Frequency Optomechanical Characterization of a Silicon Nitride Drum, Sci. Rep. 10, 1654 (2020).

[34] D. Allan, Statistics of Atomic Frequency Standards, Proc. IEEE 54, 221 (1966).
[35] S. Ranković, Y.-C. Liang, and R. Renner, Quantum Clocks and Their Synchronisation-The Alternate Ticks Game, arXiv:1506.01373.

[36] M. Brunelli et al., Experimental Determination of Irreversible Entropy Production in Out-of-Equilibrium Mesoscopic Quantum Systems, Phys. Rev. Lett. 121, 160604 (2018).

[37] Y. Guryanova, N. Friis, and M. Huber, Ideal Projective Measurements Have Infinite Resource Costs, Quantum 4, 222 (2020).

[38] L. Gammaitoni, P. Hänggi, P. Jung, and F. Marchesoni, Stochastic Resonance, Rev. Mod. Phys. 70, 223 (1998).

[39] P. Pietzonka, F. Ritort, and U. Seifert, Finite-Time Generalization of the Thermodynamic Uncertainty Relation, Phys. Rev. E 96, 012101 (2017).

[40] W. H. Press, S. A. Teukolsky, W. T. Vetterling, and B. P. Flannery, Numerical Recipes: The Art of Scientific Computing (Cambridge University Press, Cambridge, England, 2007).

[41] A. A. Clerk, M. H. Devoret, S. M. Girvin, F. Marquardt, and R. J. Schoelkopf, Introduction to Quantum Noise, Measurement, and Amplification, Rev. Mod. Phys. 82, 1155 (2010). 